\title{
ON ARBITRAGE AND DUALITY UNDER MODEL UNCERTAINTY AND PORTFOLIO CONSTRAINTS ${ }^{12}$
}

\author{
Erhan Bayraktar ${ }^{\ddagger 1}$ and Zhou Zhou ${ }^{\S 2}$ \\ University of Michigan
}

\begin{abstract}
We consider the fundamental theorem of asset pricing (FTAP) and the hedging prices of options under non-dominated model uncertainty and portfolio constraints in discrete time. We first show that no arbitrage holds if and only if there exists some family of probability measures such that any admissible portfolio value process is a local super-martingale under these measures. We also get the non-dominated optional decomposition with constraints. From this decomposition, we obtain the duality of the super-hedging prices of European options, as well as the sub- and superhedging prices of American options. Finally, we get the FTAP and the duality of super-hedging prices in a market where stocks are traded dynamically and options are traded statically.
\end{abstract}

KEY WORDS. Fundamental theorem of asset pricing, sub-(super-)hedging, model uncertainty, portfolio constraints, optional decomposition.

\section{INTRODUCTION}

We consider the fundamental theorem of asset pricing (FTAP) and the hedging prices of European and American options under the non-dominated model certainty framework of [5] with convex closed portfolio constraints in discrete time. We first show that no arbitrage in the quasi-sure sense is equivalent to the existence of a set of probability measures; under each of these measures any admissible portfolio value process is a local super-martingale. Then, we get the non-dominated version of the optional decomposition under portfolio constraints. From this optional decomposition, we get the duality of super- and sub-hedging prices of European and American options. We also show that the optimal super-hedging strategies exist. Finally, we add options to the market and get the FTAP and the duality of super-hedging prices of European options by using semi-static trading strategies (i.e., strategies dynamically trading in stocks and statically trading in options).

Our results generalize the ones in [8, Section 9] to a non-dominated model-uncertainty set-up, and extend the results in [5] to the case where portfolio constraints are involved. These conclusions are general enough to cover many interesting models with the so-called delta constraints; for example, when shorting stocks is not allowed, or some stocks enter or leave the market at certain times.

Compared to [8, Section 9], the main difficulty in our setting is due to the fact that the set of probability measures does not admit a dominating measure. We use the measurable selection

\footnotetext{
${ }^{1}$ This version: April 13, 2015; First version: February 11, 2014.

${ }^{2}$ This research is supported in part by the National Science Foundation under grant DMS-0955463.

${ }^{\ddagger}$ Department of Mathematics, University of Michigan, erhan@umich.edu.

$\S^{\S}$ Department of Mathematics, University of Michigan, zhouzhou@umich.edu.
} typesetting, pagination and proofreading process, which may lead to differences between this version and the Version of Record. Please cite this article as doi: $10.1111 /$ mafi.12104

This article is protected by copyright. All rights reserved. 
mechanism developed in [5] to overcome this difficulty, i.e., first establish the FTAP and superhedging result in one period, and then "measurably" glue all the periods together to derive multipleperiod versions. It is therefore of crucial importance to get the one-period results. In [5], Lemma 3.3 serves as a fundamental tool to show the FTAP and super-hedging result in a one-period model, whose proof uses an induction on the number of stocks and a separating hyperplane argument. In our set-up, both of these arguments do not work due to the presence of constraints. In this paper, we instead use a finite covering argument to overcome the difficulty stemming from constraints. Another major difference with [5] is the proof for the existence of an optimal super-hedging strategy in multiple periods. In [5], the existence is a direct consequence of Theorem 2.2. A key step in the proof of Theorem 2.2 is modifying the trading strategy to the one with smaller "rank" which yet still gives the same portfolio value. However, this approach fails to work in our set-up, because the modified strategy may not be admissible anymore due to the portfolio constraints. In our paper, we first find an optimal static option trading strategy, and then determine an optimal dynamic stock trading strategy using the optional decomposition theorem with constraints. The optional decomposition theorem also helps us obtain the duality results for the American options.

We work within the no-arbitrage framework of [5], in which an arbitrage is said to exist when there exists a trading strategy whose gain is quasi surely non-negative and strictly positive with positive probability under an admissible measure. In this framework, we are given a model and the non-dominated set of probability measures comes from estimating the parameters of the model. As estimating results in confidence intervals for the parameters, we end up with a set of non-dominated probability measures.

There is another no-arbitrage framework, which was introduced by Acciaio et al. [1]. In that framework, an arbitrage is said to exist if the gain from trading is strictly positive for all scenarios. Under the framework of [1], the model uncertainty is in fact part of the model itself and the user of that model does not have confidence in her ability to estimate the parameters. The choice between the frameworks of [1] and [5] is a modeling issue.

Our assumptions mainly consist of two parts: (1) the closedness and convexity of the related control sets (see Assumptions 2.1, 3.1, 4.1 and 5.1), and (2) some measurability assumptions (see the set-up of Section 3.1 and Assumptions 3.1 and 5.1). The first part is almost necessary (see Example 2.2), and can be easily verified in many interesting cases (see e.g., Example 2.1). The second part is the analyticity of some relevant sets, which we make in order to apply measurable selection results and perform dynamic programming principle type of arguments. Analyticity (which is a measurability concept more general than Borel measurability, so in particular every Borel set is analytic) is a minimal assumption needed in order to have a dynamic programming principle and this goes well back to Blackwell. These concepts are covered by standard textbooks on measure theory, see e.g. [6]. See also [4] for applications in stochastic control theory and the references therein. In Section 3.3, we provide some general and easily verifiable sufficient conditions for Assumptions 3.1(iii) and 5.1(ii), as well as Examples 3.1 and 3.2.

The rest of the paper is organized as follows: We show the FTAP in one period and in multiple periods in Sections 2 and 3 respectively. In Section 4, we get the super-hedging result in one period. 
In Section 5, we provide the non-dominated optional decomposition with constraints in multiple periods. Then, starting from the optional decomposition, we analyze the sub- and super-hedging prices of European and American options in multiple periods in Section 6. In Section 7, we add options to the market, and study the FTAP and super-hedging using semi-static trading strategies in multiple periods. Finally in the appendix, we provide the proofs of Lemmas 3.1, 3.2, 5.1 and 5.3; these proofs contain a lot of technicalities and can be safely skipped at the first reading.

We devote the rest of this section to frequently used notation and concepts in the paper.

\subsection{Frequently used notation and concepts.}

- $\mathfrak{P}(\Omega)$ denotes the set of all the probability measures on $(\Omega, \mathcal{B}(\Omega))$, where $\Omega$ is some polish space, and $\mathcal{B}(\Omega)$ denotes its Borel $\sigma$-algebra. $\mathfrak{P}(\Omega)$ is endowed with the topology of weak convergence.

- $\Delta S_{t}(\omega, \cdot)=S_{t+1}(\omega, \cdot)-S_{t}(\omega), \omega \in \Omega_{t}:=\Omega^{t}$ (t-fold Cartesian product of $\Omega$ ). We may simply write $\Delta S$ when there is only one period (i.e., $t=0$ ).

- Let $\mathcal{P} \subset \mathfrak{P}\left(\Omega_{t}\right)$. A property holds $\mathcal{P}-$ q.s. if and only if it holds $P$-a.s. for any $P \in \mathcal{P}$. A set $A \in \Omega_{t}$ is $\mathcal{P}$-polar if $\sup _{P \in \mathcal{P}} P(A)=0$.

- Let $\mathcal{P} \subset \mathfrak{P}(\Omega)$. $\operatorname{supp}_{\mathcal{P}}(\Delta S)$ is defined as the smallest closed subset $A \subset \mathbb{R}^{d}$ such that $\Delta S \in A \mathcal{P}-$ q.s.. Define $N(\mathcal{P}):=\left\{H \in \mathbb{R}^{d}: H \Delta S=0, \mathcal{P}-q . s.\right\}$ and $N^{\perp}(\mathcal{P}):=$ $\operatorname{span}\left(\operatorname{supp}_{\mathcal{P}}(\Delta S)\right) \subset \mathbb{R}^{d}$. Then $N^{\perp}(\mathcal{P})=(N(\mathcal{P}))^{\perp}$ by $[9$, Lemma 2.6]. Denote $N(P)=$ $N(\{P\})$ and $N^{\perp}(P)=N^{\perp}(\{P\})$.

- For $\mathcal{H} \subset \mathbb{R}^{d}, \mathcal{H}(\mathcal{P}):=\operatorname{proj}_{N^{\perp}(\mathcal{P})} \mathcal{H}$, where proj is short for projection. Denote $\mathcal{H}(P)=$ $\mathcal{H}(\{P\})$.

- For $\mathcal{H} \subset \mathbb{R}^{d}, \mathcal{C}_{\mathcal{H}}(\mathcal{P}):=\{c H: H \in \mathcal{H}(\mathcal{P}), c \geq 0\}$. Denote $\mathcal{C}_{\mathcal{H}}(P)=\mathcal{C}_{\mathcal{H}}(\{P\})$.

- $\mathfrak{C}_{\mathcal{H}}:=\left\{c H \in \mathbb{R}^{d}: H \in \mathcal{H}, c \geq 0\right\}$, where $\mathcal{H} \subset \mathbb{R}^{d}$.

- $(H \cdot S)_{t}=\sum_{i=0}^{t-1} H_{i}\left(S_{i+1}-S_{i}\right)$.

- $\mathbb{R}^{*}:=[-\infty, \infty]$.

- $\|\cdot\|$ represents the Euclidean norm.

- $E_{P}|X|:=E_{P}\left|X^{+}\right|-E_{P}\left|X^{-}\right|$, and by convention $\infty-\infty=-\infty$. Similarly the conditional expectation is also defined in this extended sense.

- $L_{+}^{0}(\mathcal{P})$ is the space of random variables $X$ on the corresponding topological space satisfying $X \geq 0 \mathcal{P}$-q.s., and $L^{1}(\mathcal{P})$ is the space of random variables $X$ satisfying $\sup _{P \in \mathcal{P}} E_{P}|X|<\infty$. Denote $L_{+}^{0}(P)=L_{+}^{0}(\{P\})$, and $L^{1}(P)=L^{1}(\{P\})$. Similar definitions apply for $L^{0}, L_{+}^{1}$ and $L^{\infty}$. We shall sometimes omit $\mathcal{P}$ or $P$ in $L_{+}^{0}, L^{1}$, etc., when there is no ambiguity.

- We say $\mathrm{NA}(\mathcal{P})$ holds, if for any $H \in \mathcal{H}$ satisfying $(H \cdot S)_{T} \geq 0 \mathcal{P}$ - q.s., we have that $(H \cdot S)_{T}=0 \mathcal{P}-q . s$. , where $\mathcal{H}$ is some admissible control set of trading strategies for stocks. Let $\mathrm{NA}(P)$ stand for $\mathrm{NA}(\{P\})$.

- We write $Q \lll \mathcal{P}$, if there exists some $P \in \mathcal{P}$ such that $Q \ll P$.

- Let $(X, \mathcal{G})$ be a measurable space and $Y$ be a topological space. A mapping $\Phi$ from $X$ to the power set of $Y$ is denoted by $\Phi: X \rightarrow Y$. We say $\Phi$ is measurable (resp. Borel

This article is protected by copyright. All rights reserved. 
measurable), if

$$
\{x \in X: \Phi(x) \cap A \neq \emptyset\} \in \mathcal{G}, \forall \text { closed (resp. Borel measurable) } A \subset Y \text {. }
$$

$\Phi$ is closed (resp. compact) valued if $\Phi(x) \subset Y$ is closed (resp. compact) for all $x \in X$. We refer to [2, Chapter 18] for these concepts.

- A set of random variables $A$ is $\mathcal{P}$ - q.s. closed, if $\left(a_{n}\right)_{n} \subset A$ convergent to some $a \mathcal{P}-$ q.s. implies $a \in A$.

- For $\Phi: X \rightarrow Y, \operatorname{graph}(\Phi):=\{(x, y) \in X \times Y: y \in \Phi(x)\}$.

- Let $X$ be a Polish space. A set $A \subset X$ is analytic if it is the image of a Borel subset of another Polish space under a Borel measurable mapping. A function $f: X \mapsto \mathbb{R}^{*}$ is upper (resp. lower) semianalytic if the set $\{f>c\}$ (resp. $\{f<c\}$ ) is analytic. The acronym "u.s.a." (resp. "l.s.a.") is short for upper (resp. lower) semianalytic.

- Let $X$ be a polish space. The $\sigma$-algebra $\cap_{P \in \mathfrak{P}(X)} \mathcal{B}(X)^{P}$ is called the universal completion of $\mathcal{B}(X)$, where $\mathcal{B}(X)^{P}$ is the $P$-completion of $\mathcal{B}(X)$. A set $A \subset X$ is universally measurable if $A \in \cap_{P \in \mathfrak{P}(X)} \mathcal{B}(X)^{P}$. A function $f$ is universally measurable if $f \in \cap_{P \in \mathfrak{P}(X)} \mathcal{B}(X)^{P}$. "u.m." is short for universally measurable.

- Let $X$ and $Y$ be some Borel spaces and $U: X \rightarrow Y$. Then $u$ is a u.m. selector of $U$, if $u: X \mapsto Y$ is u.m. and $u(\cdot) \in U(\cdot)$ on $\{U \neq \emptyset\}$.

\section{The FTAP IN ONE PERIOD}

In this section, we derive the FTAP for one-period model in this section. Theorem 2.1 is the main result.

2.1. The set-up and the main result. Let $\mathcal{P}$ be a convex set of probability measures on a Polish space $\Omega$. Let $S_{0} \in \mathbb{R}^{d}$ be the initial stock price, and Borel measurable $S_{1}: \Omega \mapsto \mathbb{R}^{d}$ be the stock price at time $t=1$. Denote $\Delta S=S_{1}-S_{0}$. Let $\mathcal{H} \subset \mathbb{R}^{d}$ be the set of admissible trading strategies. We assume that $\mathcal{H}$ satisfies the following conditions:

Assumption 2.1. $\mathcal{C}_{\mathcal{H}}(\mathcal{P})$ is (i) convex, and (ii) closed.

Example 2.1. Let $\mathcal{H}:=\prod_{i=1}^{d}\left[\underline{a}^{i}, \bar{a}^{i}\right]$ for some $\underline{a}^{i}, \bar{a}^{i} \in \mathbb{R}$ with $\underline{a}^{i} \leq \bar{a}^{i}, i=1 \ldots, d$. Then $\mathcal{H}$ satisfies Assumption 2.1 for any $\mathcal{P} \subset \mathfrak{P}(\Omega)$. Indeed, $\mathcal{H} \subset \mathbb{R}^{d}$ is a bounded, closed and convex set with finitely many vertices, and so is $\mathcal{H}(\mathcal{P})$. Hence the generated cone $\mathcal{C}_{\mathcal{H}}(\mathcal{P})$ is convex and closed.

Define

$$
\mathcal{Q}:=\left\{Q \in \mathfrak{P}(\Omega): Q \lll \mathcal{P}, E_{Q}|\Delta S|<\infty \text { and } E_{Q}[H \Delta S] \leq 0, \forall H \in \mathcal{H}\right\}
$$

The following is the main result of this section:

Theorem 2.1. Let Assumption 2.1 hold. Then $N A(\mathcal{P})$ holds if and only if for any $P \in \mathcal{P}$, there exists $Q \in \mathcal{Q}$ dominating $P$. 
2.2. Proof for Theorem 2.1. Let us first prove the following lemma, which is the simplified version of Theorem 2.1 when $\mathcal{P}$ consists of a single probability measure.

Lemma 2.1. Let $P \in \mathfrak{P}(\Omega)$ and Assumption 2.1 w.r.t. $\mathcal{C}_{\mathcal{H}}(P)$ hold. Then $N A(P)$ holds if and only if there exists $Q \sim P$, such that $E_{Q}|\Delta S|<\infty$ and $E_{Q}[H \Delta S] \leq 0$, for any $H \in \mathcal{H}$.

Proof. Sufficiency is obvious. We shall prove the necessity in two steps. Without loss of generality we assume that $E_{P}|\Delta S|<\infty$ (see e.g., [5, Lemma 3.2]).

Step 1: In this step, we will show that $K-L_{+}^{0}$ is closed in $L^{0}$, where

$$
K:=\left\{H \Delta S: H \in \mathcal{C}_{\mathcal{H}}(P)\right\}
$$

Let $X_{n}=H_{n} \Delta S-Y_{n} \stackrel{P}{\rightarrow} X$, where $H_{n} \in \mathcal{C}_{\mathcal{H}}(P)$ and $Y_{n} \geq 0$. Without loss of generality, assume that $X_{n} \rightarrow X, P$-a.s.. If $\left(H_{n}\right)_{n}$ is not bounded, then let $0<\left\|H_{n_{k}}\right\| \rightarrow \infty$ and we have that

$$
\frac{H_{n_{k}}}{\left\|H_{n_{k}}\right\|} \Delta S=\frac{X_{n_{k}}}{\left\|H_{n_{k}}\right\|}+\frac{Y_{n_{k}}}{\left\|H_{n_{k}}\right\|} \geq \frac{X_{n_{k}}}{\left\|H_{n_{k}}\right\|} .
$$

Taking the limits on both sides along a further sub-sequence, we obtain that $H \Delta S \geq 0 P$-a.s. for some $H \in \mathbb{R}^{d}$ with $\|H\|=1$. As $\mathcal{C}_{\mathcal{H}}(P)$ is closed, $H \Delta S \in \mathcal{C}_{\mathcal{H}}(P)$. $\operatorname{By~} \mathrm{NA}(P), H \Delta S=0$-a.s., which implies that $H \in N(P) \cap N^{\perp}(P)=\{0\}$. This contradicts $\|H\|=1$. Therefore, $\left(H_{n}\right)_{n}$ is bounded, and thus there exists a subsequence $\left(H_{n_{j}}\right)_{j}$ convergent to some $H^{\prime} \in \mathcal{C}_{\mathcal{H}}(P)$. Then

$$
0 \leq Y_{n_{j}}=H_{n_{j}} \Delta S-X_{n_{j}} \rightarrow H^{\prime} \Delta S-X=: Y, \quad P \text {-a.s.. }
$$

Then $X=H^{\prime} \Delta S-Y \in K-L_{+}^{0}$.

Step 2: From Step 1, we know that $K^{\prime}:=\left(K-L_{+}^{0}\right) \cap L^{1}$ is a closed and convex cone in $L^{1}$, and contains $-L_{+}^{\infty}$. Moreover, by $\mathrm{NA}(P), K^{\prime} \cap L_{+}^{1}=\{0\}$. Then the Kreps-Yan theorem (see e.g., [8, Theorem 1.61]) implies the existence of $Q \sim P$ with $d Q / d P \in L_{+}^{\infty}(P)$, such that $E_{Q}[H \Delta S] \leq 0$ for any $H \in \mathcal{H}$.

Remark 2.1. The FTAP under a single probability measure with constraints is analyzed in [8, Chapter 9]. However, although the idea is quite insightful, the result there is not correct: what we need is the closedness of the generated cone $\mathcal{C}_{\mathcal{H}}(P)$, instead of the closedness of $\mathcal{H}(P)$. (In this sense, our result is different from [7]; in [7] it is the closedness of the corresponding projection that matters.) Below is a counter-example to [8, Theorem 9.9].

Example 2.2. Consider the one-period model: there are two stocks $S^{1}$ and $S^{2}$ with the path space $\{(1,1)\} \times\{(s, 0): s \in[1,2]\}$; let

$$
\mathcal{H}:=\left\{\left(h_{1}, h_{2}\right): h_{1}^{2}+\left(h_{2}-1\right)^{2} \leq 1\right\} .
$$

be the set of admissible trading strategies; let $P$ be a probability measure on this path space such that $S_{1}^{1}$ is uniformly distributed on $[1,2]$. It is easy to see that $\mathrm{NA}(P)$ holds, and $\mathcal{H}$ satisfies the assumptions (a), (b) and (c) on [8, page 350]. Let $H=\left(h_{1}, h_{2}\right)$ such that $H \Delta S=0, P$-a.s. Then $h_{1}\left(S_{1}^{1}-1\right)=h_{2}, P$-a.s., which implies $h_{1}=h_{2}=0$. By [8, Remark 9.1], $\mathcal{H}$ also satisfies assumption (d) on [8, page 350].

This article is protected by copyright. All rights reserved. 
If $[8$, Theorem 9.9] were to be true, then there would exist $Q \sim P$, such that

$$
E_{Q}[H \Delta S] \leq 0, \quad \forall H \in \mathcal{H} .
$$

As $Q \sim P, E_{Q}\left(S_{1}^{1}-1\right)>0$. Take $\left(h_{1}, h_{2}\right) \in \mathcal{H}$ with $h_{1}, h_{2}>0$ and $h_{2} / h_{1}<E_{Q}\left(S_{1}^{1}-1\right)$. Then

$$
h_{1} E_{Q}\left(S_{1}^{1}-1\right)-h_{2}>0,
$$

which would contradict (2.1).

In fact, it is not hard to see that in this example,

$$
\mathcal{C}_{\mathcal{H}}(P)=\left\{\left(h_{1}, h_{2}\right): h_{2}>0 \text { or } h_{1}=h_{2}=0\right\}
$$

is not closed.

Lemma 2.2. Let Assumption 2.1(ii) hold. Then there exists $P^{\prime \prime} \in \mathcal{P}$, such that $N^{\perp}\left(P^{\prime \prime}\right)=N^{\perp}(\mathcal{P})$ and $N A\left(P^{\prime \prime}\right)$ holds.

Proof. Denote $\mathbb{H}:=\left\{H \in \mathcal{C}_{\mathcal{H}}(\mathcal{P}):\|H\|=1\right\}$. For any $H \in \mathbb{H} \subset N^{\perp}(\mathcal{P})$, by $\mathrm{NA}(\mathcal{P})$ there exists $P_{H} \in \mathcal{P}$, such that $P_{H}(H \Delta S<0)>0$. It can be further shown that there exists $\varepsilon_{H}>0$, such that for any $H^{\prime} \in B\left(H, \varepsilon_{H}\right)$,

$$
P_{H}\left(H^{\prime} \Delta S<0\right)>0,
$$

where $B\left(H, \varepsilon_{H}\right):=\left\{H^{\prime \prime} \in \mathbb{R}^{d}:\left\|H^{\prime \prime}-H\right\|<\varepsilon_{H}\right\}$. Indeed, there exists some $\delta>0$ such that $P_{H}(H \Delta S<-\delta)>0$. Then, there exists some $M>0$, such that $P_{H}(H \Delta S<-\delta,\|\Delta S\|<M)>0$. Taking $\varepsilon_{H}:=\delta / M$, we have that for any $H^{\prime} \in B\left(H, \varepsilon_{H}\right), P_{H}\left(H^{\prime} \Delta S<0,\|\Delta S\|<M\right)>0$, which implies (2.2).

Because $\mathbb{H} \subset \cup_{H \in \mathbb{H}} B\left(H, \varepsilon_{H}\right)$ and $\mathbb{H}$ is compact from Assumption 2.1, there exists a finite cover of $\mathbb{H}$, i.e., $\mathbb{H} \subset \cup_{i=1}^{n} B\left(H_{i}, \varepsilon_{H_{i}}\right)$. Let $P^{\prime}=\sum_{i=1}^{n} a_{i} P_{H_{i}}$, with $\sum_{i=1}^{n} a_{i}=1$ and $a_{i}>0, i=1, \ldots, n$. Then $P^{\prime} \in \mathcal{P}$, and $P^{\prime}(H \Delta S<0)>0$ for any $H \in \mathbb{H}$.

Obviously, $N^{\perp}\left(P^{\prime}\right) \subset N^{\perp}(\mathcal{P})$. If $N^{\perp}\left(P^{\prime}\right)=N^{\perp}(\mathcal{P})$, then let $P^{\prime \prime}=P^{\prime}$. Otherwise, take $H \in$ $N^{\perp}(\mathcal{P}) \cap N\left(P^{\prime}\right)$. Then, there exists $R_{1} \in \mathcal{P}$, such that $R_{1}(H \Delta S \neq 0)>0$. Let $R_{1}^{\prime}=\left(P^{\prime}+R_{1}\right) / 2$. Then $P^{\prime} \ll R_{1}^{\prime} \in \mathcal{P}$, and thus $N^{\perp}\left(R_{1}^{\prime}\right) \supset N^{\perp}\left(P^{\prime}\right)$. As $H \in N\left(P^{\prime}\right) \backslash N\left(R_{1}^{\prime}\right)$, we have that $N^{\perp}\left(R_{1}^{\prime}\right) \supsetneqq$ $N^{\perp}\left(P^{\prime}\right)$. If $N^{\perp}\left(R_{1}^{\prime}\right) \varsubsetneqq N^{\perp}(\mathcal{P})$, then we can similarly construct $R_{2}^{\prime} \in \mathcal{P}$, such that $R_{2}^{\prime} \gg R_{1}^{\prime}$ and $N^{\perp}\left(R_{2}^{\prime}\right) \supsetneqq N^{\perp}\left(R_{1}^{\prime}\right)$. As $N^{\perp}(\mathcal{P})$ is a finite dimensional vector space, after finite such steps, we can find such $P^{\prime \prime} \in \mathcal{P}$ dominating $P^{\prime}$ with $N^{\perp}\left(P^{\prime \prime}\right)=N^{\perp}(\mathcal{P})$. For any $H \in \mathbb{H}, P^{\prime \prime}(H \Delta S<0)>0$ because $P^{\prime \prime} \gg P^{\prime}$. This implies that $\mathrm{NA}\left(P^{\prime \prime}\right)$ holds.

Proof of Theorem 2.1. Sufficiency. If the conclusion were not true, then there would exist $H \in$ $\mathcal{H}$ and $P \in \mathcal{P}$, such that $H \Delta S \geq 0 P$-a.s., and $P(H \Delta S>0)>0$. Take $Q \in \mathcal{Q}$ with $Q \gg P$. Then $E_{Q}[H \Delta S] \leq 0$, which would contradict $H \Delta S \geq 0 Q$-a.s. and $Q(H \Delta S>0)>0$.

Necessity. Take $P \in \mathcal{P}$. By Lemma 2.2 there exists $P^{\prime \prime} \in \mathcal{P}$ such that $N^{\perp}\left(P^{\prime \prime}\right)=N^{\perp}(\mathcal{P})$ and $\mathrm{NA}\left(P^{\prime \prime}\right)$ holds. Let $R:=\left(P+P^{\prime \prime}\right) / 2 \in \mathcal{P}$. Then $N^{\perp}(R)=N^{\perp}\left(P^{\prime \prime}\right)=N^{\perp}(\mathcal{P})$, and thus $\mathcal{C}_{\mathcal{H}}(R)=\mathcal{C}_{\mathcal{H}}(\mathcal{P})$ which is convex and closed by Assumption 2.1. Besides, $\mathrm{NA}\left(P^{\prime \prime}\right)$ implies that for any $H \in \mathcal{C}_{\mathcal{H}}(R) \backslash\{0\}=\mathcal{C}_{\mathcal{H}}\left(P^{\prime \prime}\right) \backslash\{0\}, P^{\prime \prime}(H \Delta S<0)>0$, and thus $R(H \Delta S<0)>0$ as $R \gg P^{\prime \prime}$. This shows that $\mathrm{NA}(R)$ holds. From Lemma 2.1, there exists $Q \sim R \gg P$, such that $E_{Q}|\Delta S|<\infty$ and $E_{Q}[H \Delta S] \leq 0$ for any $H \in \mathcal{H}$. 


\section{The FTAP IN MULTiPle PeRiods}

In this section, we derive the FTAP in multiple periods. We will reduce it to a one-step problem and apply Theorem 2.1. Theorem 3.1 is the main result of this section.

3.1. The set-up and the main result. We use the set-up in [5]. Let $T \in \mathbb{N}$ be the time horizon and let $\Omega$ be a Polish space. For $t \in\{0,1, \ldots, T\}$, let $\Omega_{t}:=\Omega^{t}$ be the $t$-fold Cartesian product, with the convention that $\Omega_{0}$ is a singleton. We denote by $\mathcal{F}_{t}$ the universal completion of $\mathcal{B}\left(\Omega_{t}\right)$, and we shall often treat $\Omega_{t}$ as a subspace of $\Omega_{T}$. For each $t \in\{0, \ldots, T-1\}$ and $\omega \in \Omega_{t}$, we are given a nonempty convex set $\mathcal{P}_{t}(\omega) \subset \mathfrak{P}(\Omega)$ of probability measures. Here, $\mathcal{P}_{t}$ represents the possible models for the $t$-th period, given state $\omega$ at time $t$. We assume that for each $t$, the graph of $\mathcal{P}_{t}$ is analytic, which ensures by the Jankov-von Neumann Theorem (see, e.g., [4, Proposition 7.49]) that $\mathcal{P}_{t}$ admits a u.m. selector, i.e., a u.m. kernel $P_{t}: \Omega_{t} \rightarrow \mathfrak{P}(\Omega)$ such that $P_{t}(\omega) \in \mathcal{P}_{t}(\omega)$ for all $\omega \in \Omega_{t}$. Let

$$
\mathcal{P}:=\left\{P_{0} \otimes \ldots \otimes P_{T-1}: P_{t}(\cdot) \in \mathcal{P}_{t}(\cdot), t=0, \ldots, T-1\right\},
$$

where each $P_{t}$ is a u.m. selector of $\mathcal{P}_{t}$, and

$$
P_{0} \otimes \ldots \otimes P_{T-1}(A)=\int_{\Omega} \ldots \int_{\Omega} 1_{A}\left(\omega_{1}, \ldots, \omega_{T}\right) P_{T-1}\left(\omega_{1}, \ldots, \omega_{T-1} ; d \omega_{T}\right) \ldots P_{0}\left(d \omega_{1}\right), \quad A \in \Omega_{T} .
$$

Let $S_{t}=\left(S_{t}^{1}, \ldots, S_{t}^{d}\right): \Omega_{t} \rightarrow \mathbb{R}^{d}$ be Borel measurable, which represents the price at time $t$ of a stock $S$ that can be traded dynamically in the market.

For each $t \in\{0, \ldots, T-1\}$ and $\omega \in \Omega_{t}$, we are given a set $\mathcal{H}_{t}(\omega) \subset \mathbb{R}^{d}$, which is thought of as the set of admissible controls for the $t$-th period, given state $\omega$ at time $t$. We assume for each $t$, graph $\left(\mathcal{H}_{t}\right)$ is analytic, and thus admits a u.m. selector; that is, an $\mathcal{F}_{t}$-measurable function $H_{t}(\cdot): \Omega_{t} \mapsto \mathbb{R}^{d}$, such that $H_{t}(\omega) \in \mathcal{H}_{t}(\omega)$. We introduce the set of admissible portfolio controls $\mathcal{H}:$

$$
\mathcal{H}:=\left\{\left(H_{t}\right)_{t=0}^{T-1}: H_{t} \text { is a u.m. selector of } \mathcal{H}_{t}, t=0, \ldots, T-1\right\} .
$$

Then, for any $H \in \mathcal{H}, H$ is an adapted process. We make the following assumptions on $\mathcal{H}$.

\section{Assumption 3.1.}

(i) $0 \in \mathcal{H}_{t}(\omega)$, for $\omega \in \Omega_{t}, t=0, \ldots, T-1$.

(ii) $\mathcal{C}_{\mathcal{H}_{t}(\omega)}\left(\mathcal{P}_{t}(\omega)\right)$ is closed and convex, for $\omega \in \Omega_{t}, t=0, \ldots, T-1$.

(iii) The set

$$
\Psi_{\mathcal{H}_{t}}:=\left\{(\omega, Q) \in \Omega_{t} \times \mathfrak{P}(\Omega): E_{Q}\left|\Delta S_{t}(\omega, \cdot)\right|<\infty \text { and } E_{Q}\left[y \Delta S_{t}(\omega, \cdot)\right] \leq 0, \forall y \in \mathcal{H}_{t}(\omega)\right\}
$$

is analytic, for $t=0, \ldots, T-1$.

\footnotetext{
${ }^{4}$ To avoid burdening the reader with further notation we prefer to use the same notation $\mathcal{P}$ for the set of probability measures in one-period models and multi-period models. We will do the same for other sets of probability measures that appear later in the paper and also for the set of admissible strategies.
}

This article is protected by copyright. All rights reserved. 
Define

$$
\begin{aligned}
\mathcal{Q}:= & \left\{Q \in \mathfrak{P}\left(\Omega_{T}\right): Q \lll \mathcal{P}, E_{Q}\left[\left|\Delta S_{t}\right| \mid \mathcal{F}_{t}\right]<\infty Q \text {-a.s. } t=0, \ldots, T-1,\right. \\
& H \cdot S \text { is a } Q \text {-local-supermartingale } \forall H \in \mathcal{H}\} .
\end{aligned}
$$

Below is the main theorem of this section:

Theorem 3.1. Under Assumption 3.1, NA(P) holds if and only if for each $P \in \mathcal{P}$, there exists $Q \in \mathcal{Q}$ dominating $P$.

3.2. Proof of Theorem 3.1. We first provide some auxiliary results. The following lemma essentially says that if there is no arbitrage in $T$ periods, then there is no arbitrage in any period. It is parallel to [5, Lemma 4.6]. Our proof mainly focuses on the difference due to the presence of constraints and we put the proof in the appendix.

Lemma 3.1. Let $t \in\{0, \ldots, T-1\}$. Then the set

$$
N_{t}:=\left\{\omega \in \Omega_{t}: N A\left(\mathcal{P}_{t}(\omega)\right) \text { fails }\right\}
$$

is u.m., and if Assumption 3.1(i) and $N A(\mathcal{P})$ hold, then $N_{t}$ is $\mathcal{P}$-polar.

The lemma below is a measurable version of Theorem 2.1. It is parallel to [5, Lemma 4.8]. We provide its proof in the appendix.

Lemma 3.2. Let $t \in\{0, \ldots, T-1\}$, let $P(\cdot): \Omega_{t} \mapsto \mathfrak{P}(\Omega)$ be Borel, and let $\mathcal{Q}_{t}: \Omega_{t} \rightarrow \mathfrak{P}(\Omega)$,

$$
\mathcal{Q}_{t}(\omega):=\left\{Q \in \mathfrak{P}(\Omega): Q \lll \mathcal{P}_{t}(\omega), E_{Q}\left|\Delta S_{t}(\omega, \cdot)\right|<\infty, E_{Q}\left[y \Delta S_{t}(\omega, \cdot)\right] \leq 0, \forall y \in \mathcal{H}_{t}(\omega)\right\} .
$$

If Assumption 3.1(ii)(iii) holds, then $\mathcal{Q}_{t}$ has an analytic graph and there exist u.m. mappings $Q(\cdot), \hat{P}(\cdot): \Omega_{t} \rightarrow \mathfrak{P}(\Omega)$ such that

$$
\begin{aligned}
& P(\omega) \ll Q(\omega) \ll \hat{P}(\omega) \quad \text { for all } \omega \in \Omega_{t}, \\
& \hat{P}(\omega) \in \mathcal{P}_{t}(\omega) \quad \text { if } P(\omega) \in \mathcal{P}_{t}(\omega), \\
& Q(\omega) \in \mathcal{Q}_{t}(\omega) \quad \text { if } N A\left(\mathcal{P}_{t}(\omega)\right) \text { holds and } P(\omega) \in \mathcal{P}_{t}(\omega) .
\end{aligned}
$$

Proof of Theorem 3.1. Using Lemmas 3.1 and 3.2, we can perform the same glueing argument Bouchard and Nutz use in the proof of [5, Theorem 4.5], and thus we omit it here.

3.3. Sufficient conditions for Assumption 3.1(iii). By [4, Proposition 7.47], the map $(\omega, Q) \mapsto$ $\sup _{y \in \mathcal{H}_{t}(\omega)} E_{Q}\left[y \Delta S_{t}(\omega, \cdot)\right]$ is u.s.a., which does not necessarily imply the analyticity of $\Psi_{\mathcal{H}_{t}}$ as the complement of an analytic set may fail to be analytic. Therefore we provide some sufficient conditions for Assumption 3.1(iii) below.

Definition 3.1. We call $\mathfrak{H}_{t}: \Omega_{t} \rightarrow \mathbb{R}^{d}$ a stretch of $\mathcal{H}_{t}$, if for any $\omega \in \Omega_{t}, \mathfrak{C}_{\mathfrak{H}_{t}(\omega)}=\mathfrak{C}_{\mathcal{H}_{t}(\omega)}$.

It is easy to see that for any stretch $\mathfrak{H}_{t}$ of $\mathcal{H}_{t}$,

$$
\Psi_{\mathcal{H}_{t}}=\Psi_{\mathfrak{H}_{t}}=\left\{(\omega, Q) \in \Omega_{t} \times \mathfrak{P}(\Omega): E_{Q}\left|\Delta S_{t}(\omega, \cdot)\right|<\infty, \sup _{y \in \mathfrak{H}_{t}(\omega)} y E_{Q}\left[\Delta S_{t}(\omega, \cdot)\right] \leq 0\right\} .
$$

This article is protected by copyright. All rights reserved. 
Therefore, in order to show $\Psi_{\mathcal{H}_{t}}$ is analytic, it suffices to show that there exists a stretch $\mathfrak{H}_{t}$ of $\mathcal{H}_{t}$, such that the map $\varphi_{\mathfrak{H}_{t}}: \Omega_{t} \times \mathfrak{P}(\Omega) \mapsto \mathbb{R}^{*}$

$$
\varphi_{\mathfrak{H}_{t}}(\omega, Q)=\sup _{y \in \mathfrak{H}_{t}(\omega)} y E_{Q}\left[\Delta S_{t}(\omega, \cdot)\right]
$$

is 1.s.a. on $\mathcal{J}:=\left\{(\omega, Q) \in \Omega_{t} \times \mathfrak{P}(\Omega): E_{Q}\left|\Delta S_{t}(\omega, \cdot)\right|<\infty\right\}$.

Proposition 3.1. If there exists a measurable (w.r.t. $\mathcal{B}\left(\mathbb{R}^{d}\right)$ ) stretch $\mathfrak{H}_{t}$ of $\mathcal{H}_{t}$ with nonempty compact values, then $\varphi_{\mathfrak{H}_{t}}$ is Borel measurable, and thus $\Psi_{\mathcal{H}_{t}}$ is Borel measurable.

Proof. The conclusion follows directly from [2, Theorem 18.19].

Proposition 3.2. If there exists a stretch $\mathfrak{H}_{t}$ of $\mathcal{H}_{t}$ satisfying

(i) $\operatorname{graph}\left(\mathfrak{H}_{t}\right)$ is Borel measurable,

(ii) there exists a countable set $\left(y_{n}\right)_{n} \subset \mathbb{R}^{d}$, such that for any $\omega \in \Omega_{t}$ and $y \in \mathfrak{H}_{t}(\omega)$, there exists $\left(y_{n_{k}}\right)_{k} \subset\left(y_{n}\right)_{n} \cap \mathfrak{H}_{t}$ converging to $y$,

then $\varphi_{\mathfrak{H}_{t}}$ is Borel measurable, and thus $\Psi_{\mathcal{H}_{t}}$ is Borel measurable.

Proof. Define function $\phi: \mathbb{R}^{d} \times \mathcal{J} \mapsto \mathbb{R}^{*}$,

$$
\phi(y, \omega, Q)=\left\{\begin{array}{cl}
y E_{Q}\left[\Delta S_{t}(\omega, \cdot)\right] & \text { if } y \in \mathfrak{H}_{t}(\omega) \\
-\infty & \text { otherwise }
\end{array}\right.
$$

It can be shown by a monotone class argument that $\phi$ is Borel measurable. So the function $\varphi: \mathcal{J} \mapsto \mathbb{R}$

$$
\varphi(\omega, Q)=\sup _{n} \phi\left(y_{n}, \omega, Q\right)
$$

is Borel measurable. It remains to show that $\varphi=\varphi_{\mathfrak{H}_{t}}$. It is easy to see that $\varphi \geq \varphi_{\mathfrak{H}_{t}}$. Conversely, take $(\omega, Q) \in \mathcal{J}$. Then $\phi\left(y_{n}, \omega, Q\right)=y_{n} E_{Q}[\Delta S(\omega, \cdot)] \leq \varphi_{\mathfrak{H}_{t}}(\omega, Q)$ if $y_{n} \in \mathfrak{H}_{t}(\omega)$, and $\phi\left(y_{n}, \omega, Q\right)=$ $-\infty<\varphi_{\mathfrak{H}_{t}}(\omega, Q)$ if $y_{n} \notin \mathfrak{H}_{t}(\omega)$; i.e., $\varphi(\omega, Q)=\sup _{n} \phi\left(y_{n}, \omega, Q\right) \leq \varphi_{\mathfrak{H}_{t}}(\omega, Q)$.

Example 3.1. Let $\underline{a}_{t}^{i}, \bar{a}_{t}^{i}: \Omega_{t} \mapsto \mathbb{R}$ be Borel measurable, with $\underline{a}_{t}^{i}<\bar{a}_{t}^{i}, i=1, \ldots, d$. Let

$$
\mathcal{H}_{t}(\omega)=\prod_{i=1}^{d}\left[\underline{a}_{t}^{i}(\omega), \bar{a}_{t}^{i}(\omega)\right], \quad \omega \in \Omega_{t}
$$

Then both Propositions 3.1 and 3.2 hold with $\mathfrak{H}_{t}=\mathcal{H}_{t}$ and $\left(y_{n}\right)_{n}=\mathbb{Q}^{d}$.

Example 3.2. Let $d=1$ and $\mathcal{H}_{t}$ be such that for any $\omega \in \Omega_{t}, \mathcal{H}_{t}(\omega) \subset(0, \infty)$. We assume that $\operatorname{graph}\left(\mathcal{H}_{t}\right)$ is analytic, but not Borel. Then $\mathcal{H}_{t}$ itself does not satisfy the assumptions in Proposition 3.1 or 3.2. Now let $\mathfrak{H}_{t}(\omega)=[1,2], \omega \in \Omega_{t}$. Then $\mathfrak{H}_{t}$ is a stretch of $\mathcal{H}_{t}$, and $\mathfrak{H}_{t}$ satisfies the assumptions in Propositions 3.1 and 3.2 with $\left(y_{n}\right)_{n}=\mathbb{Q}$.

This article is protected by copyright. All rights reserved. 


\section{SUPER-HEDGING IN ONE PERIOD}

4.1. The set-up and the main result. We use the set-up in Section 2. Let $f$ be a u.m. function. Define the super-hedging price

$$
\pi^{\mathcal{P}}(f):=\inf \{x: \exists H \in \mathcal{H} \text {, s.t. } x+H \cdot S \geq f, \mathcal{P}-\text { q.s. }\} .
$$

We also denote $\pi^{P}(f)=\pi^{\{P\}}(f)$. We further assume:

Assumption 4.1. $\mathcal{H}(\mathcal{P})$ is convex and closed.

Remark 4.1. It is easy to see that if $\mathcal{H}(\mathcal{P})$ is convex, then $\mathcal{C}_{\mathcal{H}}(\mathcal{P})$ is convex.

Define

$$
\mathfrak{Q}:=\left\{Q \in \mathfrak{P}(\Omega): Q \lll \mathcal{P}, E_{Q}|\Delta S|<\infty, A^{Q}:=\sup _{H \in \mathcal{H}} E_{Q}[H \Delta S]<\infty\right\} .
$$

Below is the main result of this section.

Theorem 4.1. Let Assumptions 2.1(ii) \& 4.1 and $N A(\mathcal{P})$ hold. Then

$$
\pi^{\mathcal{P}}(f)=\sup _{Q \in \mathfrak{Q}}\left(E_{Q}[f]-A^{Q}\right) .
$$

Besides, $\pi^{\mathcal{P}}(f)>-\infty$ and there exists $H \in \mathcal{H}$ such that $\pi^{\mathcal{P}}(f)+H \Delta S \geq f \mathcal{P}-$ q.s..

4.2. Proof of Theorem 4.1. We first provide two lemmas.

Lemma 4.1. Let $N A(\mathcal{P})$ hold. If $\mathcal{H}(\mathcal{P})$ and $\mathcal{C}_{\mathcal{H}}(\mathcal{P})$ are closed, then

$$
\pi^{\mathcal{P}}(f)=\sup _{P \in \mathcal{P}} \pi^{P}(f) .
$$

Proof. It is easy to see that $\pi^{\mathcal{P}}(f) \geq \sup _{P \in \mathcal{P}} \pi^{P}(f)$. We prove the reverse inequality. If $\pi^{\mathcal{P}}(f)>$ $\sup _{P \in \mathcal{P}} \pi^{P}(f)$, then there exists $\varepsilon>0$ such that

$$
\alpha:=\pi^{\mathcal{P}}(f) \wedge \frac{1}{\varepsilon}-\varepsilon>\sup _{P \in \mathcal{P}} \pi^{P}(f) .
$$

By Lemma 2.2, there exists $P^{\prime \prime} \in \mathcal{P}$, such that $N^{\perp}\left(P^{\prime \prime}\right)=N^{\perp}(\mathcal{P})$ and $\mathrm{NA}\left(P^{\prime \prime}\right)$ holds.

Moreover, we have that the set

$$
A_{\alpha}:=\left\{H \in \mathcal{H}(\mathcal{P}): \alpha+H \Delta S \geq f, P^{\prime \prime}-\text { a.s. }\right\}
$$

is compact. In order to prove this claim take $\left(H_{n}\right)_{n} \subset A_{\alpha}$. Assume $\left(H_{n}\right)_{n}$ is not bounded, so up to a subsequence $0<\left\|H_{n}\right\| \rightarrow \infty$. As a result,

$$
\frac{\alpha}{\left\|H_{n}\right\|}+\frac{H_{n}}{\left\|H_{n}\right\|} \Delta S \geq \frac{f}{\left\|H_{n}\right\|} .
$$

As $\mathcal{C}_{\mathcal{H}}(\mathcal{P})$ is closed, there exist some $H \in \mathcal{C}_{\mathcal{H}}(\mathcal{P})=\mathcal{C}_{\mathcal{H}}\left(P^{\prime \prime}\right)$ with $\|H\|=1$ such that $H_{n_{k}} /\left\|H_{n_{k}}\right\| \rightarrow$ $H$. Taking the limit along $\left(n_{k}\right)_{k}$, we have $H \Delta S \geq 0 P^{\prime \prime}$-a.s.. $\mathrm{NA}\left(P^{\prime \prime}\right)$ implies that $H \Delta S=0 P^{\prime \prime}$ a.s.. Hence $H \in \mathcal{C}_{\mathcal{H}}\left(P^{\prime \prime}\right) \cap N\left(P^{\prime \prime}\right)=\{0\}$, which contradicts $\|H\|=1$. Thus $\left(H_{n}\right)_{n}$ is bounded, and there exists $H^{\prime \prime} \in \mathbb{R}^{d}$, such that $\left(H_{n_{j}}\right)_{j} \rightarrow H^{\prime \prime}$. As $\mathcal{H}(\mathcal{P})$ is closed, $H^{\prime \prime} \in \mathcal{H}(\mathcal{P})$, which further implies $H^{\prime \prime} \in A_{\alpha}$. 
For any $H \in A_{\alpha}$, as $\alpha<\pi^{\mathcal{P}}(f)$ by (4.2), there exists $P_{H} \in \mathcal{P}$ such that

$$
P_{H}(\alpha+H \Delta S<f)>0 .
$$

It can be further shown that there exists $\delta_{H}>0$, such that for any $H^{\prime} \in B\left(H, \delta_{H}\right)$,

$$
P_{H}\left(\alpha+H^{\prime} \Delta S<f\right)>0 .
$$

Because $A_{\alpha} \subset \cup_{H \in A_{\alpha}} B\left(H, \delta_{H}\right)$ and $A_{\alpha}$ is compact, there exists $\left(H_{i}\right)_{i=1}^{n} \subset A_{\alpha}$, such that $A_{\alpha} \subset$ $\cup_{i=1}^{n} B\left(H_{i}, \delta_{H_{i}}\right)$. Let

$$
P^{\prime}:=\sum_{i=1}^{n} a_{i} P_{H_{i}}+a_{0} P^{\prime \prime} \in \mathcal{P},
$$

where $\sum_{i=0}^{n} a_{i}=1$ and $a_{i}>0, i=0, \ldots, n$. Then, it is easy to see that for any $H \in \mathcal{H}(\mathcal{P})=$ $\mathcal{H}\left(P^{\prime \prime}\right)=\mathcal{H}\left(P^{\prime}\right)$,

$$
P^{\prime}(\alpha+H \Delta S<f)>0 \text {. }
$$

This implies that

$$
\alpha \leq \pi^{P^{\prime}}(f) \leq \sup _{P \in \mathcal{P}} \pi^{P}(f)
$$

which contradicts (4.2).

Lemma 4.2. Let $N A(\mathcal{P})$ hold. If $\mathcal{H}(\mathcal{P})$ and $\mathcal{C}_{\mathcal{H}}(\mathcal{P})$ are closed, then the set

$$
K(\mathcal{P}):=\left\{H \Delta S-X: H \in \mathcal{H}, X \in L_{+}^{0}(\mathcal{P})\right\}
$$

is $\mathcal{P}-$ q.s. closed.

Proof. Let $W^{n}=H^{n} \Delta S-X^{n} \in K(\mathcal{P}) \rightarrow W \mathcal{P}-$ q.s., where without loss of generality $H^{n} \in$ $\mathcal{H}(\mathcal{P})$ and $X^{n} \in L_{+}^{0}(\mathcal{P}), n=1,2, \ldots$ Assume $\left(H^{n}\right)_{n}$ is not bounded, so up to a subsequence $0<\left\|H^{n}\right\| \rightarrow \infty$. Consider

$$
\frac{W^{n}}{\left\|H^{n}\right\|}=\frac{H^{n}}{\left\|H^{n}\right\|} \Delta S-\frac{X^{n}}{\left\|H^{n}\right\|} .
$$

As $\left(H^{n} /\left\|H^{n}\right\|\right)_{n}$ is bounded, there exists some subsequence $\left(H^{n_{k}} /\left\|H^{n_{k}}\right\|\right)_{k}$ converging to some $H \in \mathbb{R}^{d}$ with $\|H\|=1$. Taking the limit in (4.5) along $\left(n_{k}\right)_{k}$, we get that $H \Delta S \geq 0 \mathcal{P}-$ q.s.. Because $\left(H^{n_{k}} /\left\|H^{n_{k}}\right\|\right)_{k} \in \mathcal{C}_{\mathcal{H}}(\mathcal{P})$ and $\mathcal{C}_{\mathcal{H}}(\mathcal{P})$ is closed, $H \in \mathcal{C}_{\mathcal{H}}(\mathcal{P})$. Hence $H \Delta S=0 \mathcal{P}-$ q.s. by $\mathrm{NA}(\mathcal{P})$. Then $H \in \mathcal{C}_{\mathcal{H}}(\mathcal{P}) \cap N(\mathcal{P})=\{0\}$, which contradicts $\|H\|=1$.

Therefore, $\left(H^{n}\right)_{n}$ is bounded and there exists some subsequence $\left(H^{n_{j}}\right)_{j}$ converging to some $H^{\prime} \in \mathbb{R}^{d}$. As $\mathcal{H}(\mathcal{P})$ is closed, $H^{\prime} \in \mathcal{H}(\mathcal{P})$. Let $X:=H^{\prime} \Delta S-W \in L_{+}^{0}(\mathcal{P})$. Then $W=H^{\prime} \Delta S-X \in$ $K(\mathcal{P})$.

Proof of Theorem 4.1. We first show that $\pi^{\mathcal{P}}(f)>-\infty$ and that an optimal super-hedging strategy exists. If $\pi^{\mathcal{P}}(f)=\infty$ then we are done. If $\pi^{\mathcal{P}}(f)=-\infty$, then for any $n \in \mathbb{N}$, there exists $H^{n} \in \mathcal{H}$ such that

$$
H^{n} \Delta S \geq f+n \geq(f+n) \wedge 1, \quad \mathcal{P}-q . s . .
$$

By Lemma 4.2, there exists some $H \in \mathcal{H}$ such that $H \Delta S \geq 1 \mathcal{P}$-q.s., which contradicts $\mathrm{NA}(\mathcal{P})$. If $\pi^{\mathcal{P}}(f) \in(-\infty, \infty)$, then for any $n \in \mathbb{N}$, there exists some $\tilde{H}^{n} \in \mathcal{H}$, such that $\pi^{\mathcal{P}}(f)+1 / n+\tilde{H}^{n} \Delta S \geq$ $f \mathcal{P}$-q.s.. Lemma 4.2 implies that there exists some $\tilde{H} \in \mathcal{H}$, such that $\pi^{\mathcal{P}}(f)+\tilde{H} \Delta S \geq f \mathcal{P}-$ q.s.. 
By Lemma 4.1,

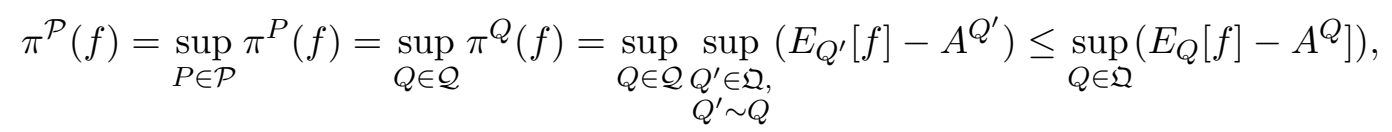

where we apply Theorem 2.1 for the second equality, and [8, Proposition 9.23] for the third equality. Conversely, if $\pi^{\mathcal{P}}(f)=\infty$, then we are done. Otherwise let $x>\pi^{\mathcal{P}}(f)$, and there exists $H \in \mathcal{H}$, such that $x+H \Delta S \geq f \mathcal{P}-$ q.s.. Then for any $Q \in \mathfrak{Q}$,

$$
x \geq E_{Q}[f]-E_{Q}[H \Delta S] \geq E_{Q}[f]-A^{Q} .
$$

By the arbitrariness of $x$ and $Q$, we have that

$$
\pi^{\mathcal{P}}(f) \geq \sup _{Q \in \mathfrak{Q}}\left(E_{Q}[f]-A^{Q}\right)
$$

which together with (4.6) implies (4.1).

\section{Optional DECOMPOSITION IN MULTIPLE PERIODS}

5.1. The set-up and the main result. We use the set-up in Section 3. In addition, let $f: \Omega_{T} \mapsto$ $\mathbb{R}$ be u.s.a. We further assume:

\section{Assumption 5.1.}

(i) For $t \in\{0, \ldots, T-1\}$ and $\omega \in \Omega_{t},\left(\mathcal{H}_{t}(\omega)\right)\left(\mathcal{P}_{t}(\omega)\right)$ is convex and closed;

(ii) the map $A_{t}(\omega, Q): \Omega_{t} \times \mathfrak{P}(\Omega) \mapsto \mathbb{R}^{*}$,

$$
A_{t}(\omega, Q)=\sup _{y \in \mathcal{H}_{t}(\omega)} y E_{Q}\left[\Delta S_{t}(\omega, \cdot)\right]
$$

is l.s.a. on the set $\left\{(\omega, Q): E_{Q}\left|\Delta S_{t}(\omega, \cdot)\right|<\infty\right\}$.

Remark 5.1. Observe that $\Psi_{\mathcal{H}_{t}}$ defined in Assumption 3.1 satisfies

$$
\Psi_{\mathcal{H}_{t}}=\left\{(\omega, Q) \in \Omega_{t} \times \mathfrak{P}(\Omega): E_{Q}\left|\Delta S_{t}(\omega, \cdot)\right|<\infty, A_{t}(\omega, Q) \leq 0\right\} .
$$

Therefore, Assumption 5.1(ii) implies Assumption 3.1(iii).

Remark 5.2. If Proposition 3.1 or 3.2 holds with $\mathfrak{H}_{t}=\mathcal{H}_{t}$, then as $A_{t}=\varphi_{\mathfrak{H}_{t}}\left(\varphi_{\mathfrak{H}_{t}}\right.$ is defined in (3.3)), Assumption 5.1(ii) holds. See Example 3.1 for a case when this holds.

For any $Q \in \mathfrak{P}\left(\Omega_{T}\right)$, there are Borel kernels $Q_{t}: \Omega_{t} \mapsto \mathfrak{P}(\Omega), t=0, \ldots, T-1$, such that $Q=Q_{0} \otimes \ldots \otimes Q_{T-1}$. For $E^{Q}\left[\left|\Delta S_{t}\right| \mid \mathcal{F}_{t}\right]<\infty Q$-a.s., define $A_{t}^{Q}(\cdot):=A_{t}\left(\cdot, Q_{t}(\cdot)\right)$ for $t=0, \ldots, T-1$, and

$$
B_{0}^{Q}:=0, \quad B_{t}^{Q}:=\sum_{i=0}^{t-1} A_{i}^{Q}, \quad t=1, \ldots, T
$$

and

$$
\mathfrak{Q}:=\left\{Q \in \mathfrak{P}\left(\Omega_{T}\right): Q \lll \mathcal{P}, E_{Q}\left[\left|\Delta S_{t}\right| \mid \mathcal{F}_{t}\right]<\infty Q \text {-a.s. for all } t \text {, and } B_{T}^{Q}<\infty Q \text {-a.s. }\right\} .
$$


Then it is not difficult to see that $\mathcal{Q} \subset \mathfrak{Q}$, where $\mathcal{Q}$ is defined in (3.1). ${ }^{5}$ Furthermore, if for each $t \in\{0, \ldots, T-1\}$ and $\omega \in \Omega_{t}, \mathcal{H}_{t}(\omega)$ is a convex cone, then $\mathfrak{Q}=\mathcal{Q}$. Below is the main result of this section.

Theorem 5.1. Let Assumptions $3.1 \& 5.1$ and $N A(\mathcal{P})$ hold. Let $V$ be an adapted process such that $V_{t}$ is u.s.a. for $t=1, \ldots, T$. Then the following are equivalent:

(i) $V-B^{Q}$ is a $Q$-local-supermartingale for each $Q \in \mathfrak{Q}$.

(ii) There exists $H \in \mathcal{H}$ and an adapted increasing process $C$ with $C_{0}=0$ such that

$$
V_{t}=V_{0}+(H \cdot S)_{t}-C_{t}, \quad \mathcal{P}-q . s .
$$

5.2. Proof of Theorem 5.1. We first provide three lemmas for the proof of Theorem 5.1. We will prove Lemmas $5.1 \& 5.3$ in the appendix.

Lemma 5.1. Let Assumption 5.1(ii) hold, and define $\mathfrak{Q}_{t}: \Omega_{t} \rightarrow \mathfrak{P}(\Omega)$ by

$$
\mathfrak{Q}_{t}(\omega):=\left\{Q \in \mathfrak{P}(\Omega): Q \lll \mathcal{P}_{t}(\omega), E_{Q}\left|\Delta S_{t}(\omega, \cdot)\right|<\infty, A_{t}(\omega, Q)<\infty\right\} .
$$

Then $\mathfrak{Q}_{t}$ has an analytic graph.

The following lemma, which is a measurable version of Theorem 4.1, is parallel to [5, Lemma 4.10]. Given Theorem 4.1, the proof of this lemma follows exactly the argument of [5, Lemma 4.10], and thus we omit it here.

Lemma 5.2. Let $N A(\mathcal{P})$ and Assumption 5.1 hold, and let $t \in\{0, \ldots, T-1\}$ and $\hat{f}: \Omega_{t} \times \Omega \mapsto \mathbb{R}^{*}$ be u.s.a.. Then

$$
\mathcal{E}_{t}(\hat{f}): \Omega_{t} \mapsto \mathbb{R}^{*}, \quad \mathcal{E}_{t}(\hat{f})(\omega):=\sup _{Q \in \mathfrak{Q}_{t}(\omega)}\left(E_{Q}[\hat{f}(\omega, \cdot)]-A_{t}(\omega, Q)\right)
$$

is u.s.a.. Besides, there exists a u.m. function $y(\cdot): \Omega_{t} \mapsto \mathbb{R}^{d}$ with $y(\cdot) \in \mathcal{H}_{t}(\cdot)$, such that

$$
\mathcal{E}_{t}(\hat{f})(\omega)+y(\omega) \Delta S_{t}(\omega, \cdot) \geq \hat{f}(\omega, \cdot) \quad \mathcal{P}_{t}(\omega)-q . s .
$$

for all $\omega \in \Omega_{t}$ such that $N A\left(\mathcal{P}_{t}(\omega)\right)$ holds and $\hat{f}(\omega, \cdot)>-\infty \mathcal{P}_{t}(\omega)-q . s .$.

Lemma 5.3. Let Assumptions $3.1 \& 55.1$ and $N A(\mathcal{P})$ hold. Recall $\mathfrak{Q}_{t}$ defined in (5.2). We have that

$$
\mathfrak{Q}=\left\{Q_{0} \otimes \ldots \otimes Q_{T-1}: Q_{t}(\cdot) \text { is a u.m. selector of } \mathfrak{Q}_{t}, t=0, \ldots, T-1\right\} \text {. }
$$

\footnotetext{
${ }^{5} \mathrm{~A}$ rigorous argument is as follows. Let $Q=Q_{0} \otimes \ldots \otimes Q_{T-1} \in \mathcal{Q}$, where $Q_{t}$ is a Borel kernels, $t=0, \ldots, T-1$. It can be shown by a monotone class argument that the map $\left(\omega, y, Q^{\prime}\right) \mapsto y E_{Q^{\prime}}[\Delta S(\omega, \cdot)]$ is Borel measurable for $\left(\omega, y, Q^{\prime}\right) \in \Omega_{t} \times \mathbb{R}^{d} \times \mathfrak{P}(\Omega)$. Hence the map $(\omega, y) \mapsto y E_{Q_{t}(\omega)}[\Delta S(\omega, \cdot)]$ is Borel measurable for $(\omega, y) \in \Omega_{t} \times \mathbb{R}^{d}$. As $\operatorname{graph}\left(\mathcal{H}_{t}\right)$ is analytic, by [4, Proposition 7.50] there exists a u.m. selector $H_{t}^{n}(\cdot) \in \mathcal{H}_{t}(\cdot)$, such that

$$
A_{t}^{Q}(\omega) \wedge n-1 / n \leq H_{t}^{n}(\omega) E_{Q_{t}(\omega)}\left[\Delta S_{t}(\omega, \cdot)\right] \leq 0, \text { for } Q \text {-a.s. } \omega \in \Omega_{t},
$$

where the second inequality follows from the local-supermartingale property of $H^{n} \cdot S$ with $H^{n}=$ $\left(0, \ldots, 0, H_{t}^{n}, 0 \ldots, 0\right) \in \mathcal{H}$. Sending $n \rightarrow \infty$ we get that $A_{t}^{Q} \leq 0 Q$-a.s. for $t=0, \ldots, T-1$, and thus $Q \in \mathfrak{Q}$.
} 
Proof of Theorem 5.1. (ii) $\Longrightarrow$ (i): For any $Q \in \mathfrak{Q}$,

$$
V_{t+1}=V_{t}+H_{t} \Delta S_{t}-\left(C_{t+1}^{Q}-C_{t}^{Q}\right) \leq V_{t}+H_{t} \Delta S_{t}, Q \text {-a.s.. }
$$

Hence,

$$
E_{Q}\left[V_{t+1} \mid \mathcal{F}_{t}\right] \leq V_{t}+H_{t} E_{Q}\left[\Delta S_{t} \mid \mathcal{F}_{t}\right] \leq V_{t}+A_{t}^{Q}=V_{t}+B_{t+1}^{Q}-B_{t}^{Q}
$$

Then

$$
E_{Q}\left[V_{t+1}-B_{t+1}^{Q} \mid \mathcal{F}_{t}\right] \leq V_{t}-B_{t}^{Q} .
$$

(i) $\Longrightarrow$ (ii): We shall first show that

$$
\mathcal{E}_{t}\left(V_{t+1}\right) \leq V_{t}, \quad \mathcal{P}-q . s . .
$$

Let $Q=Q_{1} \otimes \ldots \otimes Q_{T-1} \in \mathfrak{Q}$ and $\varepsilon>0$. The map $(\omega, Q) \rightarrow E_{Q}\left[V_{t+1}(\omega, \cdot)\right]-A_{t}(\omega, Q)$ is u.s.a., and graph $\left(\mathfrak{Q}_{t}\right)$ is analytic. As a result, by [4, Proposition 7.50], there exists a u.m. selector $Q_{t}^{\varepsilon}: \Omega_{t} \mapsto \mathfrak{P}(\Omega)$, such that $Q_{t}^{\varepsilon}(\cdot) \in \mathfrak{Q}_{t}(\cdot)$ on $\left\{\mathfrak{Q}_{t} \neq \emptyset\right\}$ (whose complement is a $Q$-null set), and

$$
E_{Q_{t}^{\varepsilon}(\cdot)}\left[V_{t+1}\right]-A_{t}\left(\cdot, Q_{t}^{\varepsilon}(\cdot)\right) \geq \mathcal{E}_{t}\left(V_{t+1}\right) \wedge \frac{1}{\varepsilon}-\varepsilon, \quad Q \text {-a.s. }
$$

Define

$$
Q^{\prime}=Q_{1} \otimes \ldots \otimes Q_{t-1} \otimes Q_{t}^{\varepsilon} \otimes Q_{t+1} \otimes Q_{T-1}
$$

Then $Q^{\prime} \in \mathfrak{Q}$ by Lemma 5.3. Therefore,

$$
E_{Q^{\prime}}\left[V_{t+1}-B_{t+1}^{Q^{\prime}} \mid \mathcal{F}_{t}\right] \leq V_{t}-B_{t}^{Q^{\prime}}, \quad Q^{\prime} \text {-a.s. }
$$

Noticing that $Q=Q^{\prime}$ on $\Omega_{t}$, we have that

$$
V_{t} \geq E_{Q^{\prime}}\left[V_{t+1} \mid \mathcal{F}_{t}\right]-A_{t}^{Q^{\prime}}=E_{Q_{t}^{\varepsilon}(\cdot)}\left[V_{t+1}\right]-A_{t}\left(\cdot, Q_{t}^{\varepsilon}(\cdot)\right) \geq \mathcal{E}_{t}\left(V_{t+1}\right) \wedge \frac{1}{\varepsilon}-\varepsilon, \quad Q \text {-a.s.. }
$$

By the arbitrariness of $\varepsilon$ and $Q$, we have that (5.4) holds.

By Lemma 5.2, there exists a u.m. function $H_{t}: \Omega_{t} \mapsto \mathbb{R}^{d}$ such that

$$
\mathcal{E}_{t}\left(V_{t+1}\right)(\omega)+H_{t}(\omega) \Delta S_{t+1}(\omega, \cdot) \geq V_{t+1}(\omega, \cdot) \quad \mathcal{P}_{t}(\omega)-q . s .
$$

for $\omega \in \Omega_{t} \backslash N_{t}$. Fubini's theorem and (5.4) imply that

$$
V_{t}+H_{t} \Delta S_{t} \geq V_{t+1} \quad \mathcal{P}-q . s .
$$

Finally, by defining $C_{t}:=V_{0}+(H \cdot S)_{t}-V_{t}$, the conclusion follows.

\section{Hedging European and American options in multiple periods}

6.1. Hedging European options. Let $f: \Omega_{T} \mapsto \mathbb{R}$ be a u.s.a. function, which represents the payoff of a European option. Define the super-hedging price

$$
\pi(f):=\inf \left\{x: \exists H \in \mathcal{H} \text {, s.t. } x+(H \cdot S)_{T} \geq f, \mathcal{P}-\text { q.s. }\right\} .
$$

Theorem 6.1. Let Assumptions $3.1 \& 5.1$ and $N A(\mathcal{P})$ hold. Then the super-hedging price is given by

$$
\pi(f)=\sup _{Q \in \mathfrak{Q}}\left(E_{Q}[f]-E_{Q}\left[B_{T}^{Q}\right]\right) .
$$

Moreover, $\pi(f)>-\infty$ and there exists $H \in \mathcal{H}$, such that $\pi(f)+(H \cdot S)_{T} \geq f \mathcal{P}-$ q.s.. 
Proof. It is easy to see that $\pi(f) \geq \sup _{Q \in \mathfrak{Q}}\left(E_{Q}[f]-E_{Q}\left[B_{T}^{Q}\right]\right)$. We show the reverse inequality. Define $V_{T}=f$ and

$$
V_{t}=\mathcal{E}_{t}\left(V_{t+1}\right), t=0, \ldots, T-1 .
$$

Then $V_{t}$ is u.s.a. by Lemma 5.2 for $t=1, \ldots, T$. It is easy to see that $\left(V_{t}-B_{t}^{Q}\right)_{t}$ is a $Q$-localsupermartingale for each $Q \in \mathfrak{Q}$. Then, by Theorem 5.1, there exists $H \in \mathcal{H}$, such that

$$
V_{0}+(H \cdot S)_{T} \geq V_{T}=f, \quad \mathcal{P}-q . s .
$$

Hence $V_{0} \geq \pi(f)$. It remains to show that

$$
V_{0} \leq \sup _{Q \in \mathfrak{Q}}\left(E_{Q}[f]-E_{Q}\left[B_{T}^{Q}\right]\right)
$$

First assume that $f$ is bounded from above. Then by [4, Proposition 7.50], Lemmas 5.1 and 5.2, we can choose a u.m. $\varepsilon$ optimizer $Q_{t}^{\varepsilon}$ for $\mathcal{E}_{t}$ in each time period. Define $Q^{\varepsilon}:=Q_{0}^{\varepsilon} \otimes \ldots \otimes Q_{T-1}^{\varepsilon} \in \mathfrak{Q}$,

$$
V_{0}=\mathcal{E}_{0} \circ \ldots \circ \mathcal{E}_{T-1}(f) \leq E_{Q^{\varepsilon}}\left[f-B_{T}^{Q^{\varepsilon}}\right]+T \varepsilon \leq \sup _{Q \in \mathfrak{Q}} E_{Q}\left[f-B_{T}^{Q}\right]+T \varepsilon
$$

which implies (6.2).

In general let $f$ be any u.s.a. function. Then, we have

$$
\mathcal{E}_{0} \circ \ldots \circ \mathcal{E}_{T-1}(f \wedge n) \leq \sup _{Q \in \mathfrak{Q}}\left(E_{Q}[f \wedge n]-E_{Q}\left[B_{T}^{Q}\right]\right)
$$

Obviously the limit of the right hand side above is $\sup _{Q \in \mathfrak{Q}}\left(E_{Q}[f]-E_{Q}\left[B_{T}^{Q}\right]\right)$. To conclude that the limit of the left hand side is $\mathcal{E}_{0} \circ \ldots \circ \mathcal{E}_{T-1}(f)$, it suffices to show that for any $t \in\{0, \ldots, T-1\}$, and $\mathcal{F}_{t+1}$-measurable functions $v^{n} \nearrow v$,

$$
\gamma:=\sup _{n} \mathcal{E}_{t}\left(v^{n}\right)=\mathcal{E}_{t}(v), \mathcal{P}-q . s .
$$

Indeed, for $\omega \in \Omega_{t} \backslash N_{t}$, by Theorem $4.1 v^{n}(\omega)-\gamma(\omega) \in K(\mathcal{P}(\omega))$, where $N_{t}$ and $K(\cdot)$ are defined

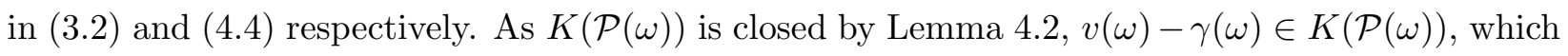
implies $\gamma(\omega) \geq \mathcal{E}_{t}(v)(\omega)$ by Theorem 4.1 .

Finally, using a backward induction, we can show that $V_{t}>-\infty \mathcal{P}-$ q.s., $t=0, \ldots, T-1$ by Lemma 3.1 and Theorem 4.1. In particular, $\pi(f)=V_{0}>-\infty$.

Corollary 6.1. Let Assumption 5.1 and $N A(\mathcal{P})$ hold. Assume that for any $t \in\{0, \ldots, T-1\}$ and $\omega \in \Omega_{t}, \mathcal{H}_{t}(\omega)$ is a convex cone containing the origin. Then

$$
\pi(f)=\sup _{Q \in \mathcal{Q}} E_{Q}[f]
$$

Proof. This follows from (5.1) and that $\mathfrak{Q}=\mathcal{Q}$ and $B_{T}^{Q}=0$ for any $Q \in \mathcal{Q}$. 
6.2. Hedging American options. We consider the sub- and super-hedging prices of an American option in this subsection. The same problems are analyzed in [3] but without portfolio constraints. The analysis here is essentially the same, so we only provide the results and the main ideas for their proofs. For more details and discussion see [3].

For $t \in\{0, \ldots, T-1\}$ and $\omega \in \Omega_{t}$, define

$$
\mathfrak{Q}^{t}(\omega):=\left\{Q_{t}(\omega) \otimes \ldots \otimes Q_{T-1}(\omega, \cdot): Q_{i} \text { is a u.m. selector of } \mathfrak{Q}_{i}, i=t, \ldots, T-1\right\} .
$$

In particular $\mathfrak{Q}^{0}=\mathfrak{Q}$. Assume that graph $\left(\mathfrak{Q}^{t}\right)$ is analytic. Let $\mathcal{T}$ be the set of stopping times with respect to the raw filtration $\left(\mathcal{B}\left(\Omega_{t}\right)\right)_{t}$, and let $\mathcal{T}_{t} \subset \mathcal{T}$ be the set of stopping times that are no less than $t$.

Let $\mathfrak{f}=\left(\mathfrak{f}_{t}\right)_{t}$ be the payoff of the American option. Assume that $\mathfrak{f}_{t} \in \mathcal{B}\left(\Omega_{t}\right), t=1, \ldots, T$, and that $\mathfrak{f}_{\tau} \in L^{1}(Q)$ for any $\tau \in \mathcal{T}$ and $Q \in \mathfrak{Q}$. Define the sub-hedging price:

$$
\underline{\pi}(\mathfrak{f}):=\sup \left\{x: \exists(H, \tau) \in \mathcal{H} \times \mathcal{T} \text {, s.t. } \mathfrak{f}_{\tau}+(H \cdot S)_{\tau} \geq x, \mathcal{P}-q . s .\right\},
$$

and the super-hedging price:

$$
\bar{\pi}(\mathfrak{f}):=\inf \left\{x: \exists H \in \mathcal{H} \text {, s.t. } x+(H \cdot S)_{\tau} \geq \mathfrak{f}_{\tau}, \mathcal{P}-\text { q.s., } \forall \tau \in \mathcal{T}\right\} .
$$

Proposition 6.1. (i) The sub-hedging price is given by

$$
\underline{\pi}(\mathfrak{f})=\sup _{\tau \in \mathcal{T}} \inf _{Q \in \mathfrak{Q}} E_{Q}\left[\mathfrak{f}_{\tau}+B_{T}^{Q}\right] .
$$

(ii) Assume that for each $t \in\{1, \ldots, T-1\}$ the map

$$
\phi_{t}: \Omega_{t} \times \mathfrak{P}\left(\Omega_{T-t}\right) \mapsto \mathbb{R}^{*}, \phi_{t}(\omega, Q)=\sup _{\tau \in \mathcal{T}_{t}} E_{Q}\left[\mathfrak{f}_{\tau}(\omega, \cdot)-\sum_{i=t}^{\tau-1} A_{i}^{Q}(\omega, \cdot)\right]
$$

is u.s.a.. Then

$$
\bar{\pi}(\mathfrak{f})=\sup _{\tau \in \mathcal{T}} \sup _{Q \in \mathfrak{Q}} E_{Q}\left[\mathfrak{f}_{\tau}-B_{\tau}^{Q}\right]
$$

and there exists $H \in \mathcal{H}$, such that $\bar{\pi}(f)+(H \cdot S)_{\tau} \geq \mathfrak{f}_{\tau}, \mathcal{P}-q . s ., \forall \tau \in \mathcal{T}$.

Proof. (i) We first show that

$$
\underline{\pi}(f)=\sup \left\{x: \exists(H, \tau) \in \mathcal{H} \times \mathcal{T} \text {, s.t. } \mathfrak{f}_{\tau}+(H \cdot S)_{T} \geq x, \mathcal{P}-\text { q.s. }\right\}=: \beta .
$$

For any $x<\underline{\pi}(f)$, there exists $(H, \tau) \in \mathcal{H} \times \mathcal{T}$, such that $\mathfrak{f}_{\tau}+(H \cdot S)_{\tau} \geq x \mathcal{P}-q . s$.. Define $H^{\prime}:=\left(H_{t} 1_{\{t<\tau\}}\right)_{t}$. For $t=0, \ldots, T-1$, as $\{t<\tau\} \in \mathcal{B}\left(\Omega_{t}\right), H_{t}^{\prime}(\cdot)$ is u.m.; besides, $H_{t}^{\prime}(\cdot)$ is equal to either $H_{t}(\cdot) \in \mathcal{H}_{t}(\cdot)$ or $0 \in \mathcal{H}_{t}(\cdot)$. Hence $H^{\prime} \in \mathcal{H}$. Then $\mathfrak{f}_{\tau}+\left(H^{\prime} \cdot S\right)_{T}=\mathfrak{f}_{\tau}+(H \cdot S)_{\tau} \geq x \mathcal{P}-q . s$, which implies $x \leq \beta$, and thus $\underline{\pi}(f) \leq \beta$.

Conversely, for $x<\beta$, there exists $(H, \tau) \in \mathcal{H} \times \mathcal{T}$, such that $\mathfrak{f}_{\tau}+(H \cdot S)_{T} \geq x \mathcal{P}-q . s$.. Then we also have that $\mathfrak{f}_{\tau}+(H \cdot S)_{\tau} \geq x \mathcal{P}-q . s$. To see this, let us define $D:=\left\{\mathfrak{f}_{\tau}+(H \cdot S)_{\tau}<x\right\}$ and $H^{\prime}:=\left(H_{t} 1_{\{t \geq \tau\} \cap D}\right)_{t} \in \mathcal{H}$. We get that

$$
\left(H^{\prime} \cdot S\right)_{T}=\left[(H \cdot S)_{T}-(H \cdot S)_{\tau}\right] 1_{D} \geq 0 \mathcal{P}-q . s ., \text { and }\left(H^{\prime} \cdot S\right)_{T}>0 \mathcal{P}-\text { q.s. on } D .
$$

$\mathrm{NA}(\mathcal{P})$ implies that $D$ is $\mathcal{P}$-polar. Therefore $x \leq \underline{\pi}(f)$, and thus $\beta \leq \underline{\pi}(f)$. 
It can be shown that

$$
\underline{\pi}(\mathfrak{f})=\beta=\sup _{\tau \in \mathcal{T}} \sup \left\{x: \exists H \in \mathcal{H}: \mathfrak{f}_{\tau}+(H \cdot S)_{T} \geq x, \mathcal{P}-q . s .\right\}=\sup _{\tau \in \mathcal{T}} \inf _{Q \in \mathfrak{Q}} E_{Q}\left[\mathfrak{f}_{\tau}+B_{T}^{Q}\right],
$$

where we apply Theorem 6.1 for the last equality above.

(ii) Let $V_{T}:=\mathfrak{f}_{T}$. For $t=0, \ldots, T-1$, define $V_{t}: \Omega_{t} \mapsto \mathbb{R}^{*}$,

$$
V_{t}(\omega):=\sup _{Q \in \mathfrak{Q}^{t}} \sup _{\tau \in \mathcal{T}_{t}} E_{Q}\left[\mathfrak{f}_{\tau}(\omega, \cdot)-\sum_{i=t}^{\tau-1} A_{i}^{Q}(\omega, \cdot)\right], \quad \omega \in \Omega_{t} .
$$

It can be shown that $V_{t}$ is u.s.a. for $t=1, \ldots, T$ and $\left(V_{t}-B_{t}^{Q}\right)_{t}$ is a $Q$-supermartingale for each $Q \in \mathfrak{Q}$. By Theorem 5.1, there exists $H \in \mathcal{H}$ such that

$$
V_{0}+(H \cdot S)_{\tau} \geq \mathfrak{f}_{\tau}, \mathcal{P}-q . s ., \forall \tau \in \mathcal{T}
$$

Therefore, $\sup _{\tau \in \mathcal{T}} \sup _{Q \in \mathfrak{Q}} E_{Q}\left[\mathfrak{f}_{\tau}-B_{\tau}^{Q}\right]=V_{0} \leq \bar{\pi}(\mathfrak{f})$. The reverse inequality is easy to see.

Remark 6.1. In (6.3) and (6.4), the penalization terms are $B_{T}^{Q}$ and $B_{\tau}^{Q}$ respectively. Similar to the argument in (i) above, one can show that

$$
\begin{aligned}
\hat{\pi}(f) & :=\inf \left\{x: \forall \tau \in \mathcal{T}, \exists H \in \mathcal{H}, \text { s.t. } x+(H \cdot S)_{\tau} \geq \mathfrak{f}_{\tau}, \mathcal{P}-q . s .\right\} \\
& =\sup _{\tau \in \mathcal{T}} \inf \left\{x: \exists H \in \mathcal{H}, \text { s.t. } x+(H \cdot S)_{\tau} \geq \mathfrak{f}_{\tau}, \mathcal{P}-q . s .\right\} \\
& =\sup _{\tau \in \mathcal{T}} \inf \left\{x: \exists H \in \mathcal{H}, \text { s.t. } x+(H \cdot S)_{T} \geq \mathfrak{f}_{\tau}, \mathcal{P}-q . s .\right\} \\
& =\sup _{\tau \in \mathcal{T}} \sup _{Q \in \mathfrak{Q}} E_{Q}\left[\mathfrak{f}_{\tau}-B_{T}^{Q}\right] .
\end{aligned}
$$

Even though the definition of $\hat{\pi}(f)$ is less useful for super-hedging (because the stopping time should not be known in advance), it suggests that $B_{T}^{Q}$ comes from knowing $\tau$ in advance (compare $\underline{\pi}(f)$ and $\hat{\pi}(f))$. It is also both mathematically and financially meaningful that $\hat{\pi}(f) \leq \bar{\pi}(f)$. However, it is interesting that when $B^{Q}$ vanishes (e.g., when $\mathcal{H}_{t}(\cdot)$ is a cone), we have that $\hat{\pi}(f)=\bar{\pi}(f)$.

\section{FTAP AND SUPER-HEDGING IN MULTIPLE PERIODS WITH OPTIONS}

Let us use the set-up in Section 3. In addition, let $g=\left(g^{1}, \ldots, g^{e}\right): \Omega_{T} \mapsto \mathbb{R}^{e}$ be Borel measurable, and each $g^{i}$ is seen as an option which can only be traded at time $t=0$ without constraints. Without loss of generality, we assume that the price of each option is 0 . In this section, we say $\operatorname{NA}(\mathcal{P})^{g}$ holds if for any $(H, h) \in \mathcal{H} \times \mathbb{R}^{e}$,

$$
(H \cdot S)_{T}+h g \geq 0 \mathcal{P}-q . s . \Longrightarrow(H \cdot S)_{T}+h g=0 \mathcal{P}-q . s . .
$$

Obviously $\mathrm{NA}(\mathcal{P})^{g}$ implies $\mathrm{NA}(\mathcal{P})$.

Definition 7.1. $f: \Omega_{T} \mapsto \mathbb{R}$ is replicable (by stocks and options), if there exists some $x \in \mathbb{R}, h \in$ $\mathbb{R}^{e}$ and $H \in \mathcal{H}$, such that

$$
x+(H \cdot S)_{T}+h g=f \quad \text { or } \quad x+(H \cdot S)_{T}+h g=-f .
$$


Let

$$
\mathcal{Q}_{g}:=\left\{Q \in \mathcal{Q}: E_{Q}[g]=0\right\} .
$$

Below is the main result of this section:

Theorem 7.1. Let assumptions in Corollary 6.1 hold. Furthermore, assume that $g^{i}$ is not replicable by stocks and other options, and that $g^{i} \in L^{1}(\mathcal{Q}), i=1, \ldots, e$. Then we have the following.

(i) $N A(\mathcal{P})^{g}$ holds if and only if for each $P \in \mathcal{P}$, there exists $Q \in \mathcal{Q}_{g}$ dominating $P$.

(ii) Let $N A(\mathcal{P})^{g}$ hold. Let $f: \Omega_{T} \mapsto \mathbb{R}$ be Borel measurable such that $f \in L^{1}(\mathcal{Q})$. Then

$$
\pi(f):=\inf \left\{x \in \mathbb{R}: \exists(H, h) \in \mathcal{H} \times \mathbb{R}^{e} \text { s.t. } x+(H \cdot S)_{T}+h g \geq f, \mathcal{P}-\text { q.s. }\right\}=\sup _{Q \in \mathcal{Q}_{g}} E_{Q}[f] .
$$

Moreover, there exists $(H, h) \in \mathcal{H} \times \mathbb{R}^{e}$, such that $\pi(f)+(H \cdot S)_{T}+h g \geq f \mathcal{P}-$ q.s..

(iii) Assume in addition $\mathcal{H}=-\mathcal{H}$. Let $N A(\mathcal{P})^{g}$ hold and $f: \Omega_{T} \mapsto \mathbb{R}$ be Borel measurable satisfying $f \in L^{1}\left(\mathcal{Q}_{g}\right)$. Then the following are equivalent:

(a) $f$ is replicable;

(b) The mapping $Q \mapsto E_{Q}[f]$ is a constant on $\mathcal{Q}_{g}$;

(c) For any $P \in \mathcal{P}$ there exists $Q \in \mathcal{Q}_{g}$ such that $P \ll Q$ and $E_{Q}[f]=\pi(f)$.

Moreover, the market is complete ${ }^{6}$ if and only if $\mathcal{Q}_{g}$ is a singleton.

Proof. We first show the existence of an optimal super-hedging strategy in (ii). It can be shown that

$$
\pi(f)=\inf _{h \in \mathbb{R}^{e}} \inf \left\{x \in \mathbb{R}: \exists H \in \mathcal{H} \text { s.t. } x+(H \cdot S)_{T} \geq f-h g, \mathcal{P}-q . s .\right\}=\inf _{h \in \mathbb{R}^{e}} \sup _{Q \in \mathcal{Q}} E_{Q}[f-h g],
$$

where we apply Corollary 6.1 for the second equality above.

We claim that 0 is a relative interior point of the convex set

$$
\mathcal{I}:=\left\{E_{Q}[g]: Q \in \mathcal{Q}\right\} .
$$

If not, then there exists some $h \in \mathbb{R}^{e}$ with $h \neq 0$, such that $E_{Q}[h g] \leq 0$ for any $Q \in \mathcal{Q}$. Then the super-hedging price of $h g$ using $S, \pi^{0}(h g)$, satisfies $\pi^{0}(h g) \leq 0$ by Corollary 6.1. Hence by Theorem 6.1 there exists $H \in \mathcal{H}$, such that $(H \cdot S)_{T} \geq h g \mathcal{P}-q . s$.. As the price of $h g$ is $0, \mathrm{NA}(\mathcal{P})^{g}$ implies that

$$
(H \cdot S)_{T}-h g=0 \mathcal{P}-q . s .
$$

which contradicts the assumption that each $g^{i}$ cannot be replicated by $S$ and the other options, as $h \neq 0$. Hence we have shown that 0 is a relative interior point of $\mathcal{I}$.

Define $\phi: \mathbb{R}^{e} \mapsto \mathbb{R}$,

$$
\phi(h)=\sup _{Q \in \mathcal{Q}} E_{Q}[f-h g]
$$

and observe that

$$
\pi(f)=\inf _{h \in \mathbb{R}^{e}} \phi(h)=\inf _{h \in \operatorname{span}(\mathcal{I})} \phi(h) .
$$

\footnotetext{
${ }^{6}$ That is, for any Borel measurable function $f: \Omega_{T} \mapsto \mathbb{R}$ satisfying $f \in L_{g}^{1}(\mathcal{Q}), f$ is replicable.
} 
We will now show that there exists a compact set $\mathbb{K} \subset \operatorname{span}(\mathcal{I})$, such that

$$
\pi(f)=\inf _{h \in \mathbb{K}} \phi(h) .
$$

In order to do this, we will show that any $h$ outside a particular ball will satisfy $\phi(h) \geq \phi(0)$, which establishes the claim.

Now, as 0 is a relative interior point of $\mathcal{I}$, there exists $\gamma>0$, such that

$$
B_{\gamma}:=\{v \in \operatorname{span}(\mathcal{I}):\|v\| \leq \gamma\} \subset \mathcal{I} .
$$

Consider the ball $\mathbb{K}:=\left\{h \in \operatorname{span}(\mathcal{I}):\|h\| \leq 2 \sup _{Q \in \mathcal{Q}} E_{Q}|f| / \gamma\right\}$. Then, for any $h \in \operatorname{span}(\mathcal{I}) \backslash \mathbb{K}$, there exists $Q \in \mathcal{Q}$ such that $-h E_{Q}[g]>2 \sup _{Q \in \mathcal{Q}} E_{Q}|f|$ (pick $Q$ s.t. $E_{Q}[g]$ is in the same direction as $-h$ and lies on the circumference of $B_{\gamma}$ ). This implies that

$$
\phi(h) \geq \sup _{Q \in \mathcal{Q}} E_{Q}[-h g]-\sup _{Q \in \mathcal{Q}} E_{Q}|f|>\sup _{Q \in \mathcal{Q}} E_{Q}|f|=\phi(0) .
$$

Because such an $h$ is suboptimal, it follows that

$$
\pi(f)=\inf _{h \in \mathbb{K}} \phi(h) .
$$

Now observe that

$$
\left|\phi(h)-\phi\left(h^{\prime}\right)\right| \leq \sup _{Q \in \mathcal{Q}}\left|E_{Q}[f-h g]-E_{Q}\left[f-h^{\prime} g\right]\right| \leq \sup _{Q \in \mathcal{Q}} E\left|\left(h-h^{\prime}\right) g\right| \leq\left\|h-h^{\prime}\right\| \sup _{Q \in \mathcal{Q}} E_{Q}[\|g\|],
$$

i.e. $\phi$ is continuous (in fact Lipschitz). Hence, there exists some $h^{*} \in \mathbb{K} \subset \mathbb{R}^{e}$, such that

$\pi(f)=\inf _{h \in \mathbb{R}^{e}} \sup _{Q \in \mathcal{Q}} E_{Q}[f-h g]=\sup _{Q \in \mathcal{Q}} E_{Q}\left[f-h^{*} g\right]=\inf \left\{x \in \mathbb{R}: \exists H \in \mathcal{H}\right.$ s.t. $\left.x+H \cdot S \geq f-h^{*} g, \mathcal{P}-q . s.\right\}$.

Then, by Theorem 6.1 there exists $H^{*} \in \mathcal{H}$, such that $\pi(f)+\left(H^{*} \cdot S\right)_{T} \geq f-h^{*} g \mathcal{P}-q . s$. .

Next, let us prove (i) and (7.1) in (ii) simultaneously by induction. For $e=0$, (i) and (7.1) hold by Theorem 2.1 and Corollary 6.1. Assume for $e=k$ (i) and (7.1) hold and we consider $e=k+1$. We first consider (i). Let $\pi^{k}\left(g^{k+1}\right)$ be the super-hedging price of $g^{k+1}$ using stocks $S$ and options $g^{\prime}:=\left(g^{1}, \ldots, g^{k}\right)$. By induction hypothesis, we have

$$
\pi^{k}\left(g^{k+1}\right)=\sup _{Q \in \mathcal{Q}_{g^{\prime}}} E_{Q}\left[g^{k+1}\right]
$$

Recall that the price of $g^{k+1}$ is 0 . Then $\mathrm{NA}(\mathcal{P})^{g}$ implies $\pi^{k}\left(g^{k+1}\right) \geq 0$. If $\pi^{k}\left(g^{k+1}\right)=0$, then there exists $(H, h) \in \mathcal{H} \times \mathbb{R}^{k}$, such that $(H \cdot S)_{T}+h g^{\prime}-g^{k+1} \geq 0 \mathcal{P}-q . s$. Then by $\mathrm{NA}(\mathcal{P})^{g}$,

$$
(H \cdot S)_{T}+h g^{\prime}-g^{k+1}=0, \quad \mathcal{P}-q . s .,
$$

which contradicts the assumption that $g^{k+1}$ cannot be replicated by $S$ and $g^{\prime}$. Therefore, $\pi^{k}\left(g^{k+1}\right)>$ 0 . Similarly $\pi^{k}\left(-g^{k+1}\right)>0$. Thus, we have that

$$
\inf _{Q \in \mathcal{Q}_{g^{\prime}}} E_{Q}\left[g^{k+1}\right]<0<\sup _{Q \in \mathcal{Q}_{g^{\prime}}} E_{Q}\left[g^{k+1}\right] .
$$

Then, there exists $Q_{-}, Q_{+} \in \mathcal{Q}_{g^{\prime}}$ satisfying

$$
E_{Q_{-}}\left[g^{k+1}\right]<0<E_{Q_{+}}\left[g^{k+1}\right] .
$$


Then, for any $P \in \mathcal{P}$, let $Q \in \mathcal{Q}_{g^{\prime}}$ dominate $P$. Let

$$
Q^{\prime}:=\lambda_{-} Q_{-}+\lambda Q+\lambda_{+} Q_{+}
$$

By choosing some appropriate $\lambda_{-}, \lambda, \lambda_{+}>0$ with $\lambda_{-}+\lambda+\lambda_{+}=1$, we have $P \ll Q^{\prime} \in \mathcal{Q}_{g}$, where $g=\left(g^{1}, \ldots, g^{k+1}\right)$.

Next consider (7.1) in (ii). Denote the super-hedging price $\pi^{k}(\cdot)$ when using $S$ and $g^{\prime}$, and $\pi(\cdot)$ when using $S$ and $g$, which is consistent with the definition in (7.1). It is easy to see that

$$
\pi(f) \geq \sup _{Q \in \mathcal{Q}_{g}} E_{Q}[f]
$$

and we focus on the reverse inequality. It suffices to show that

$$
\exists Q_{n} \in \mathcal{Q}_{g^{\prime}} \text {, s.t. } E_{Q_{n}}\left[g^{k+1}\right] \rightarrow 0 \text { and } E_{Q_{n}}[f] \rightarrow \pi(f) .
$$

Indeed, if (7.5) holds, then we define

$$
Q_{n}^{\prime}:=\lambda_{-}^{n} Q_{-}+\lambda^{n} Q_{n}+\lambda_{+}^{n} Q_{+}, \quad \text { s.t. } E_{Q_{n}^{\prime}}\left[g^{k+1}\right]=0 \text {, i.e., } Q_{n}^{\prime} \in \mathcal{Q}_{g},
$$

where $Q_{+}, Q_{-}$are from $(7.3)$ and $\lambda_{-}^{n}, \lambda^{n}, \lambda_{+}^{n} \in[0,1]$ such that $\lambda_{-}^{n}+\lambda^{n}+\lambda_{+}^{n}=1$. As $E_{Q_{n}}\left[g^{k+1}\right] \rightarrow 0$, we can choose $\lambda_{ \pm}^{n} \rightarrow 0$. Then $E_{Q_{n}^{\prime}}[f] \rightarrow \pi(f)$, which implies that $\pi(f) \leq \sup _{Q \in \mathcal{Q}_{g}} E_{Q}[f]$.

Let us concentrate on proving (7.5). By a translation, we may without loss of generality assume that $\pi(f)=0$. If $(7.5)$ did not hold, then we would have that

$$
0 \notin \overline{\left\{E_{Q}\left[\left(g^{k+1}, f\right)\right]: Q \in \mathcal{Q}_{g^{\prime}}\right\}} \subset \mathbb{R}^{2} .
$$

Then, there would exist a separating vector $(y, z) \in \mathbb{R}^{2}$ with $\|(y, z)\|=1$ such that

$$
\sup _{Q \in \mathcal{Q}_{g^{\prime}}} E_{Q}\left[y g^{k+1}+z f\right]<0 .
$$

By the induction hypothesis, we would have that

$$
0>\sup _{Q \in \mathcal{Q}_{g^{\prime}}} E_{Q}\left[y g^{k+1}+z f\right]=\pi^{k}\left(y g^{k+1}+z f\right) \geq \pi\left(y g^{k+1}+z f\right)=\pi(z f) .
$$

Obviously, from the above $z \neq 0$. If $z>0$, then by positive homogeneity we would have that $\pi(f)<0$, which would contradict the assumption $\pi(f)=0$. Hence $z<0$. Take $Q^{\prime \prime} \in \mathcal{Q}_{g} \subset \mathcal{Q}_{g^{\prime}}$. Then, by (7.6), $0>E_{Q^{\prime \prime}}\left[y g^{k+1}+z f\right]=E_{Q^{\prime \prime}}[z f]$, and thus $E_{Q^{\prime \prime}}[f]>0=\pi(f)$, which would contradict (7.4).

Finally, let us prove (iii). It is easy to see that $(\mathrm{a}) \Longrightarrow(\mathrm{b}) \Longrightarrow(\mathrm{c})$. Now let (c) hold. Let $(H, h) \in \mathcal{H} \times \mathbb{R}^{e}$ such that $\pi(f)+(H \cdot S)_{T}+h g \geq f \mathcal{P}-$ q.s.. If there exists $P \in \mathcal{P}$ satisfying

$$
P\left\{\pi(f)+(H \cdot S)_{T}+h g>f\right\}>0,
$$

then by choosing a $Q \in \mathcal{Q}_{g}$ that dominates $P$, we would have that $\pi(f)>E_{Q}[f]=\pi(f)$, contradiction. Hence $\pi(f)+H \cdot S+h g=f \mathcal{P}-$ q.s., i.e., $f$ is replicable.

If the market is complete, then by letting $f=1_{A}$, we know that $Q \mapsto Q(A)$ is constant on $\mathcal{Q}$ for every $A \in \mathcal{B}(\Omega)$ by (b). As any probability measure is uniquely determined by its value on $\mathcal{B}(\Omega)$, we know that $\mathcal{Q}$ is a singleton. Conversely, if $\mathcal{Q}$ is a singleton, then (b) holds, and thus by (a) the market is complete. 


\section{A. Proofs of Some Technical Results}

\section{A.1. Proof of Lemma 3.1.}

Proof. Fix $t \in\{0, \ldots, T-1\}$ and let

$$
\Lambda^{\circ}(\omega):=\left\{y \in \mathbb{R}^{d}: y v \geq 0, \text { for all } v \in \operatorname{supp}_{\mathcal{P}(\omega)}\left(\Delta S_{t}(\omega, \cdot)\right)\right\}, \quad \omega \in \Omega_{t} .
$$

It can be easily shown that

$$
N_{t}^{c}=\left\{\omega \in \Omega_{t}: \Lambda_{\mathcal{H}}^{\circ}(\omega) \subset-\Lambda^{\circ}(\omega)\right\},
$$

where $\Lambda_{\mathcal{H}}^{\circ}=\Lambda^{\circ} \cap \mathcal{H}_{t}$. For any $P \in \mathfrak{P}\left(\Omega_{t}\right)$, by [5, (4.5)], there exists a Borel-measurable mapping $\Lambda_{P}^{\circ}: \Omega_{t} \rightarrow \mathbb{R}^{d}$ with non-empty closed values such that $\Lambda_{P}^{\circ}=\Lambda^{\circ} P$-a.s.. This implies that the $\operatorname{graph}\left(\Lambda_{P}^{\circ}\right)$ is Borel (see $[2$, Theorem 18.6]). Then it can be shown directly from the definition (1.1) that $\Lambda_{\mathcal{H}, P}^{\circ}:=\Lambda_{P}^{\circ} \cap \mathcal{H}_{t}$ is u.m.. Thanks to the closedness of $-\Lambda^{\circ}$, the set

$$
N_{t, P}^{c}=\left\{\omega: \Lambda_{\mathcal{H}, P}^{\circ}(\omega) \subset-\Lambda^{\circ}(\omega)\right\}=\cap_{y \in \mathbb{Q}^{d}}\left\{\omega: \operatorname{dist}\left(y, \Lambda_{\mathcal{H}, P}^{\circ}(\omega)\right) \geq \operatorname{dist}\left(y,-\Lambda^{\circ}(\omega)\right)\right\}
$$

is u.m. Therefore, there exists a Borel measurable set $\tilde{N}_{t, P}^{c}$, such that $\tilde{N}_{t, P}^{c}=N_{t, P}^{c}=N_{t}^{c} P$-a.s.. Thus $N_{t}^{c}$ is u.m. by [4, Lemma 7.26].

It remains to show that $N_{t}$ is $\mathcal{P}$-polar. If not, then there exists $P_{*} \in \mathcal{P}$ such that $P_{*}\left(N_{t}\right)>0$. Similar to the argument above, there exists a map $\Lambda_{*}^{\circ}: \Omega_{t} \rightarrow \mathbb{R}^{d}$ with a Borel measurable graph $\left(\Lambda_{*}^{\circ}\right)$, such that

$$
\Lambda_{*}^{\circ}=\Lambda^{\circ} P_{*} \text {-a.s.. }
$$

Let

$$
\Phi(\omega):=\left\{(y, P) \in\left(\Lambda_{*}^{\circ} \cap \mathcal{H}_{t}\right)(\omega) \times \mathcal{P}_{t}(\omega): E_{P}\left[y \Delta S_{t}(\omega, \cdot)\right]>0\right\}, \quad \omega \in \Omega_{t} .
$$

Then $N_{t}=\{\Phi \neq \emptyset\} P_{*}$-a.s. by (3.2), (A.1) and (A.2). It is easy to see that (with a slight abuse of notation)

$$
\operatorname{graph}(\Phi)=\left[\operatorname{graph}\left(\mathcal{P}_{t}\right) \times \mathbb{R}^{d}\right] \cap\left[\mathfrak{P}(\Omega) \times \operatorname{graph}\left(\Lambda_{*}^{\circ}\right)\right] \cap\left\{E_{P}\left[y \Delta S_{t}(\omega, \cdot)\right]>0\right\} \cap\left[\mathfrak{P}(\Omega) \times \operatorname{graph}\left(\mathcal{H}_{t}\right)\right]
$$

is analytic. Therefore, by the Jankov-von Neumann Theorem [4, Proposition 7.49], there exists a u.m. selector $(y, P)$ such that $(y(\cdot), P(\cdot)) \in \Phi(\cdot)$ on $\{\Phi \neq \emptyset\}$. As $N_{t}=\{\Phi \neq \emptyset\} P_{*}-$ a.s., $y$ is $P_{*}$-a.s. an arbitrage on $N_{t}$. Redefine $y=0$ on $\left\{y \notin \Lambda^{\circ} \cap \mathcal{H}_{t}\right\}$, and $P$ to be any u.m. selector of $\mathcal{P}_{t}$ on $\{\Phi=\emptyset\}$. (Here we redefine $y$ on $\left\{y \notin \Lambda^{\circ} \cap \mathcal{H}_{t}\right\}$ instead of $\{\Phi \neq \emptyset\}$ in order to make sure that $y(\cdot) \in \Lambda^{\circ}(\cdot)$ so that $\left.y \Delta S_{t} \geq 0 \mathcal{P}-q . s ..\right)$ So we have that $y(\cdot) \in \mathcal{H}_{t}(\cdot), P(\cdot) \in \mathcal{P}_{t}(\cdot)$, $y \Delta S_{t} \geq 0 \mathcal{P}-q . s .$, and

$$
P(\omega)\left\{y(\omega) \Delta S_{t}(\omega, \cdot)>0\right\}>0 \quad \text { for } P_{*} \text {-a.s. } \omega \in N_{t} .
$$

Now, let $H=\left(H_{0}, \ldots, H_{T-1}\right) \in \mathcal{H}$ satisfies

$$
H_{t}=y, \text { and } H_{s}=0, s \neq t
$$

and define

$$
P^{*}:=\left.P_{*}\right|_{\Omega_{t}} \otimes P \otimes P_{t+1} \otimes \ldots \otimes P_{T-1} \in \mathcal{P},
$$

where $P_{s}$ is any u.m. selector of $\mathcal{P}_{s}, s=t+1, \ldots, T-1$. Then $(H \cdot S)_{T} \geq 0 \mathcal{P}-$ q.s., and $P^{*}\left\{(H \cdot S)_{T}>0\right\}>0$ by $($ A.3), which contradicts $\mathrm{NA}(\mathcal{P})$. 


\section{A.2. Proof of Lemma 3.2.}

Proof. Let

$$
\Phi(\omega):=\{(R, \hat{R}) \in \mathfrak{P}(\Omega) \times \mathfrak{P}(\Omega): P(\omega) \ll R \ll \hat{R}\}, \quad \omega \in \Omega_{t},
$$

which has an analytic graph as shown in the proof of $\left[5\right.$, Lemma 4.8]. Consider $\Xi: \Omega_{t} \rightarrow$ $\mathfrak{P}(\Omega) \times \mathfrak{P}(\Omega)$,

$$
\begin{aligned}
\Xi(\omega):= & \left\{(Q, \hat{P}) \in \mathfrak{P}(\Omega) \times \mathfrak{P}(\Omega): E_{Q}\left|\Delta S_{t}(\omega, \cdot)\right|<\infty, E_{Q}\left[y \Delta S_{t}(\omega, \cdot)\right] \leq 0, \forall y \in \mathcal{H}_{t}(\omega),\right. \\
& \left.P(\omega) \ll Q \ll \hat{P} \in \mathcal{P}_{t}(\omega)\right\} .
\end{aligned}
$$

Recall the analytic set $\Psi_{\mathcal{H}_{t}}$ defined Assumption 3.1(iii). We have that

$$
\operatorname{graph}(\Xi)=\left[\Psi_{\mathcal{H}_{t}} \times \mathfrak{P}(\Omega)\right] \cap\left[\mathfrak{P}(\Omega) \times \operatorname{graph}\left(\mathcal{P}_{t}\right)\right] \cap \operatorname{graph}(\Phi)
$$

is analytic. As a result, we can apply the Jankov-von Neumann Theorem [4, Proposition 7.49] to find u.m. selectors $Q(\cdot), \hat{P}(\cdot)$ such that $(Q(\cdot), \hat{P}(\cdot)) \in \Xi(\cdot)$ on $\{\Xi \neq \emptyset\}$. We set $Q(\cdot):=\hat{P}(\cdot):=P(\cdot)$ on $\{\Xi=\emptyset\}$. By Theorem 2.1, if Assumption 3.1(ii) and $\mathrm{NA}\left(\mathcal{P}_{t}(\omega)\right)$ hold, and $P(\omega) \in \mathcal{P}_{t}(\omega)$, then $\Xi(\omega) \neq \emptyset$. So our construction satisfies the conditions in the statement of the lemma.

It remains to show that $\operatorname{graph}\left(\mathcal{Q}_{t}\right)$ is analytic. Using the same argument for $\Xi$, but omitting the lower bound $P(\cdot)$, we see that the map $\tilde{\Xi}: \Omega_{t} \rightarrow \mathfrak{P}(\Omega) \times \mathfrak{P}(\Omega)$,

$$
\begin{aligned}
\tilde{\Xi}(\omega):= & \left\{(Q, \hat{P}) \in \mathfrak{P}(\Omega) \times \mathfrak{P}(\Omega): E_{Q}\left|\Delta S_{t}(\omega, \cdot)\right|<\infty, E_{Q}\left[y \Delta S_{t}(\omega, \cdot)\right] \leq 0, \forall y \in \mathcal{H}_{t}(\omega),\right. \\
& \left.Q \ll \hat{P} \in \mathcal{P}_{t}(\omega)\right\}
\end{aligned}
$$

has an analytic graph. As $\operatorname{graph}\left(\mathcal{Q}_{t}\right)$ is the image of graph $(\tilde{\Xi})$ under the canonical projection $\Omega_{t} \times \mathfrak{P}(\Omega) \times \mathfrak{P}(\Omega) \rightarrow \Omega_{t} \times \mathfrak{P}(\Omega)$, it is also analytic.

\section{A.3. Proof of Lemma 5.1.}

Proof. Similar to the argument in [5, Lemma 4.8], we can show that the set

$$
J:=\{(P, Q) \in \mathfrak{P}(\Omega) \times \mathfrak{P}(\Omega): Q \ll P\}
$$

is Borel measurable. Thus, the map $\Xi: \Omega_{t} \rightarrow \mathfrak{P}(\Omega)$ defined by

$$
\Xi(\omega):=\left\{Q \in \mathfrak{P}(\Omega): Q \lll \mathcal{P}_{t}(\omega)\right\}
$$

has an analytic graph. Indeed, this follows by observing that graph $(\Xi)$ is the projection of the analytic set

$$
\left[\Omega_{t} \times J\right] \cap\left[\operatorname{graph}\left(\mathcal{P}_{t}\right) \times \mathfrak{P}(\Omega)\right]
$$

onto $\Omega_{t} \times \mathfrak{P}(\Omega)$. By Assumption 5.1(ii), the function $\hat{A}: \Omega_{t} \times \mathfrak{P}(\Omega) \mapsto \mathbb{R}^{*}$,

$$
\hat{A}(\omega, Q)=A(\omega, Q) 1_{\left\{E_{Q}\left|\Delta S_{t}(\omega, \cdot)\right|<\infty\right\}}+\infty 1_{\left\{E_{Q}\left|\Delta S_{t}(\omega, \cdot)\right|=\infty\right\}}
$$

is l.s.a.. As a result,

$$
\operatorname{graph}\left(\mathfrak{Q}_{t}\right)=\operatorname{graph}(\Xi) \cap\{\hat{A}<\infty\}
$$

is analytic. 


\section{A.4. Proof of Lemma 5.3.}

Proof. Denote the right-hand-side of $(5.3)$ by $\mathfrak{R}$. Let $R=Q_{0} \otimes \ldots \otimes Q_{T-1} \in \mathfrak{R}$. Without loss of generality, we can assume that $Q_{t}: \Omega_{t} \mapsto \mathfrak{P}(\Omega)$ is Borel measurable and that $Q_{t}(\cdot) \in \mathfrak{Q}_{t}(\cdot)$ on $\left\{\mathfrak{Q}_{t} \neq \emptyset\right\} Q^{t-1}:=Q_{0} \otimes \ldots \otimes Q_{t-1}$-a.s., $t=1, \ldots, T-1$. Let

$$
\Phi_{t}(\omega):=\left\{(Q, P) \in \mathfrak{P}(\Omega) \times \mathfrak{P}(\Omega): Q_{t}(\omega)=Q \ll P \in \mathcal{P}_{t}(\omega)\right\}, \quad \omega \in \Omega_{t}, t=0, \ldots, T-1 .
$$

Similar to the argument in the proof of [5, Lemma 4.8], it can be shown that $\operatorname{graph}\left(\Phi_{t}\right)$ is analytic. As a result, there exist u.m. selectors $\hat{Q}_{t}(\cdot), \hat{P}_{t}(\cdot)$, such that $\left(\hat{Q}_{t}(\cdot), \hat{P}_{t}(\cdot)\right) \in \Phi(\cdot)$ on $\left\{\Phi_{t} \neq \emptyset\right\}$.

We shall show by an induction that for $t=0, \ldots, T-1$,

$$
\Phi_{0} \neq \emptyset \text {, and }\left\{\Phi_{t}=\emptyset\right\} \text { is a } Q^{t-1} \text {-null set for } t=1, \ldots T-1,
$$

and there exists a u.m. selector of $\mathcal{P}_{t}$ which we denote by $P_{t}(\cdot): \Omega_{t} \mapsto \mathfrak{P}(\Omega)$ such that

$$
Q^{t}=\hat{Q}_{0} \otimes \ldots \otimes \hat{Q}_{t} \ll P_{0} \otimes \ldots \otimes P_{t} .
$$

Then by setting $t=T-1$, we know $R=Q^{T-1} \in \mathfrak{Q}$. It is easy to see that the above holds for $t=0$. Assume it holds for $t=k<T-1$. Then $\left\{\Phi_{k+1}=\emptyset\right\} \subset\left\{Q_{k+1}(\cdot) \notin \mathfrak{Q}_{k+1}(\cdot)\right\}$ is a $Q^{k}$-null set by Lemma 3.1 and the induction hypothesis. As a result, $\hat{Q}_{k+1}=Q_{k+1} Q^{k}$-a.s., which implies that $Q^{k+1}=\hat{Q}_{0} \otimes \ldots \otimes \hat{Q}_{k+1}$. Setting $P_{k+1}:=\hat{P}_{k+1} 1_{\{\Phi \neq \emptyset\}}+\tilde{P}_{k+1} 1_{\{\Phi=\emptyset\}}$, where $\tilde{P}_{k+1}(\cdot)$ is any u.m. selector of $\mathcal{P}_{k+1}$, we have that $P_{0} \otimes \ldots \otimes P_{k+1} \in \mathcal{P}^{k+1}$. As $Q_{k+1}(\omega) \ll P_{k+1}(\omega)$ for $Q^{k}$-a.s. $\omega \in \Omega_{k}$, together with the induction hypothesis, we have that $Q^{k+1} \ll P_{0} \otimes \ldots \otimes P_{k+1}$. Now we are done with the induction.

Conversely, for any $R \in \mathfrak{Q}$, we may write $R=Q_{0} \otimes \ldots \otimes Q_{T-1}$, where $Q_{t}: \Omega_{t} \mapsto \mathfrak{P}(\Omega)$ is some Borel kernel, $t=0, \ldots, T-1$. Then $Q_{t}(\omega) \in \mathfrak{Q}_{t}(\omega)$ for $Q^{t-1}$-a.s. $\omega \in \Omega_{t-1}$. Thanks to the analyticity of $\operatorname{graph}\left(\mathfrak{Q}_{t}\right)$, we can modify $Q_{t}(\cdot)$ on a $Q^{t-1}$-null set, such that the modification $\hat{Q}_{t}(\cdot)$ is u.m. and $\hat{Q}_{t}(\cdot) \in \mathfrak{Q}_{t}(\cdot)$ on $\left\{\mathfrak{Q}_{t} \neq \emptyset\right\}$. Doing this modification procedure for each $t$, we get that $R=\hat{Q}_{0} \otimes \ldots \otimes \hat{Q}_{T-1} \in \mathfrak{R}$.

\section{REFERENCES}

[1] B. Acciaio, M. Beiglbock, F. Penkner, And W. Schachermayer, A model-free version of the fundamental theorem of asset pricing and the super-replication theorem, (2013). To appear in Mathematical Finance. Available at arXiv:1301.5568.

[2] C. D. Aliprantis And K. C. Border, Infinite dimensional analysis. A hitchhiker's guide. 3rd ed., Berlin: Springer, 3rd ed. ed., 2006.

[3] E. Bayraktar, Y.-J. Huang, And Z. Zhou, On hedging American options under model uncertainty, to appear in the SIAM Journal on Financial Mathematics. Preprint, arXiv:1309.2982.

[4] D. P. Bertsekas And S. E. Shreve, Stochastic optimal control, vol. 139 of Mathematics in Science and Engineering, Academic Press Inc. [Harcourt Brace Jovanovich Publishers], New York, 1978. The discrete time case.

[5] B. Bouchard And M. Nutz, Arbitrage and duality in nondominated discrete-time models, to appear in the Annals of Applied Probability. Available as arXiv:1305.6008.

[6] D. L. Cohn, Measure theory, Birkhäuser Advanced Texts: Basler Lehrbücher. [Birkhäuser Advanced Texts: Basel Textbooks], Birkhäuser/Springer, New York, second ed., 2013.

This article is protected by copyright. All rights reserved. 
[7] C. Czichowsky And M. Schweizer, Closedness in the semimartingale topology for spaces of stochastic integrals with constrained integrands, in Séminaire de Probabilités XLIII, vol. 2006 of Lecture Notes in Math., Springer, Berlin, 2011, pp. 413-436.

[8] H. Föllmer And A. Schied, Stochastic finance, vol. 27 of de Gruyter Studies in Mathematics, Walter de Gruyter \& Co., Berlin, extended ed., 2004. An introduction in discrete time.

[9] M. Nutz, Utility maximization under model uncertainty in discrete time, to appear in Mathematical Finance. Available as arXiv:1307.3597.

This article is protected by copyright. All rights reserved. 